\title{
Collaborative Multi-Layer Network Coding For Hybrid Cellular Cognitive Radio Networks
}

\author{
Thesis by \\ Abdallah Jalal Moubayed \\ In Partial Fulfillment of the Requirements \\ For the Degree of \\ Masters of Science (Electrical Engineering)
}

King Abdullah University of Science and Technology, Thuwal,

Kingdom of Saudi Arabia

May, 2014 
The thesis of Abdallah Jalal Moubayed is approved by the examination committee

Committee Chairperson: Mohamed-Slim Alouini

Committee Member: Tareq Al-Naffouri

Committee Member: Ahmad Sultan

Committee Member: George Turkiyyah

King Abdullah University of Science and Technology 
Copyright (C)2014

Abdallah Jalal Moubayed

All Rights Reserved 


\section{ABSTRACT}

\section{Collaborative Multi-Layer Network Coding For Hybrid Cellular Cognitive Radio Networks}

\section{Abdallah Moubayed}

In this thesis, as an extension to [1], we propose a prioritized multi-layer network coding scheme for collaborative packet recovery in hybrid (interweave and underlay) cellular cognitive radio networks. This scheme allows the uncoordinated collaboration between the collocated primary and cognitive radio base-stations in order to minimize their own as well as each other's packet recovery overheads, thus by improving their throughput. The proposed scheme ensures that each network's performance is not degraded by its help to the other network. Moreover, it guarantees that the primary network's interference threshold is not violated in the same and adjacent cells. Yet, the scheme allows the reduction of the recovery overhead in the collocated primary and cognitive radio networks. The reduction in the cognitive radio network is further amplified due to the perfect detection of spectrum holes which allows the cognitive radio base station to transmit at higher power without fear of violating the interference threshold of the primary network. For the secondary network, simulation results show reductions of $20 \%$ and $34 \%$ in the packet recovery overhead, compared to the non-collaborative scheme, for low and high probabilities of primary packet arrivals, respectively. For the primary network, this reduction was found to be $12 \%$. Furthermore, with the use of fractional cooperation, the average recovery overhead is further 
reduced by around $5 \%$ for the primary network and around $10 \%$ for the secondary network when a high fractional cooperation probability is used. 


\section{ACKNOWLEDGEMENTS}

In the name of Allah, the most merciful, the most beneficent. First, I would like to praise Allah (swt) for giving me the power and ability to complete this work.

Second, I would like to thank Dr. Sameh Sorour for all the help he gave me and all the time he spent with me discussing ideas. His insight was crucial in my understanding of the topic of network coding. His commitment towards helping me achieve my goal of completing this thesis despite him being in another city is greatly appreciated.

Third, I would like to thank my thesis supervisors Dr. Mohamed-Slim Alouini and Dr. Tareq Al-Naffouri for guiding me throughout this project. The dedication they showed and the guidance they offered me was both inspiring and integral for my completion of this thesis. I would also like to thank my committee members Dr. Ahmed Sultan and Dr. George Turkiyyah for making the most complex ideas seem accessible and understandable through their thorough explanations and vivid examples. They have made me realize that indeed "simplicity is beauty".

Last but not least, I would like to thank my family and friends for their continuous support: my father, Jalal Moubayed, for always pushing me on to more success; my mother, Najwa Moubayed, for providing me with all the nurture I needed to perform to the best of my abilities; my sisters, Hiba Moubayed Alaya and Hamsa Moubayed, for always giving me advice and believing in my capabilities; and finally my fiance, Dana Al-Wattar, for bearing with me despite my complaints and for always driving me to be a better researcher and a better man. 


\section{TABLE OF CONTENTS}

Examination Committee Approval $\quad 2$

$\begin{array}{ll}\text { Copyright } & 3\end{array}$

$\begin{array}{ll}\text { Abstract } & 4\end{array}$

$\begin{array}{ll}\text { Acknowledgements } & 6\end{array}$

$\begin{array}{lr}\text { List of Abbreviations } & 9\end{array}$

$\begin{array}{ll}\text { List of Symbols } & 10\end{array}$

$\begin{array}{ll}\text { List of Figures } & 11\end{array}$

1 Introduction 12

1.1 Motivation . . . . . . . . . . . . . . . . . . . . . 12

1.2 Cognitive Radio Networks . . . . . . . . . . . . . . . . . . . 14

1.2 .1 Definitions . . . . . . . . . . . . . . . . . . . . . 14

1.2 .2 History . . . . . . . . . . . . . . . . . . . . . . 15

1.2 .3 Techniques . . . . . . . . . . . . . . . . . . . . . 17

1.2.4 Applications . . . . . . . . . . . . . . . . . . . . . . . . 19

1.2 .5 Challenges . . . . . . . . . . . . . . . . . . . 22

1.3 Network Coding . . . . . . . . . . . . . . . . . . . . . 23

1.3.1 Definitions . . . . . . . . . . . . . . . . . . . 24

1.3 .2 Types . . . . . . . . . . . . . . . . . . . . . 24

1.3 .3 Benefits . . . . . . . . . . . . . . . . 25

1.3.4 Applications . . . . . . . . . . . . . . . . . . . 26

1.4 Thesis Organization . . . . . . . . . . . . . . . . . . . . . 27

2 Collaborative Multi-Layer Network Coding For Hybrid Cellular Cognitive Radio Networks $\quad 28$

2.1 Previous Work . . . . . . . . . . . . . . . . . . . . 28 
2.2 System Model . . . . . . . . . . . . . . . . . . . . . . . . . . . . 30

2.3 Multi-Layer IDNC Graph and Algorithm . . . . . . . . . . . . . . 31

2.3.1 Graph Theory Basics . . . . . . . . . . . . . . . . . . . . . 31

2.3.2 Clique Selection Algorithm . . . . . . . . . . . . . . . . 32

2.3.3 Multi-layer IDNC Algorithm . . . . . . . . . . . . . . . 33

2.4 Proposed IDNC-based Packet Recovery Algorithm . . . . . . . . . . 36

2.5 Simulation Results . . . . . . . . . . . . . . . . . . . . . . . 39

3 Effect of Fractional Cooperation in Collaborative Multi-Layer Network Coding in Multi-Cell Cognitive Radio Networks 46

3.1 Previous Work . . . . . . . . . . . . . . . . . . 46

3.2 System Model and Multi-Layer IDNC Graph and Algorithm . . . . . 47

3.3 Proposed IDNC-based Packet Recovery Algorithm . . . . . . . . . . 48

3.4 Simulation Results . . . . . . . . . . . . . . . . . . . . 53

4 Concluding Remarks $\quad 59$

4.1 Summary . . . . . . . . . . . . . . . . . . . . . . . 59

4.2 Future Research Work . . . . . . . . . . . . . . . . . 60

$\begin{array}{ll}\text { References } & 61\end{array}$

$\begin{array}{ll}\text { Appendices } & 67\end{array}$ 


\section{LIST OF ABBREVIATIONS}

\begin{tabular}{ll}
\hline Symbol & Meaning \\
\hline CR & Cognitive Radio \\
PU & Primary User \\
SU & Secondary User \\
PN & Primary Network \\
CRN & Cognitive Radio Network \\
RNC & Random Network Coding \\
ARQ & Automatic Repeat Request \\
IDNC & Instantly Decodable Network Coding \\
PBS & Primary Base Station \\
CBS & Cognitive Base Station \\
SDR & Software Defined Radio \\
RKRL & Radio Knowledge Representation Language \\
CSI & Channel-State Information \\
RF & Radio Frequency \\
PDA & Personal Digital Assistant \\
QoE & Quality of Experience \\
FC & Fractional Cooperation \\
\hline
\end{tabular}




\section{LIST OF SYMBOLS}

\begin{tabular}{ll}
\hline Symbol & Meaning \\
\hline$M_{P}$ & number of primary users \\
$M_{S}$ & number of secondary users \\
$N_{P}$ & number of primary packets \\
$N_{S}$ & number of secondary packets \\
$p_{i}^{(p)}$ & erasure probability for primary user $i$ \\
$p_{i}^{(s)}$ & erasure probability for secondary user $i$ \\
$U_{i}$ & User $i$ \\
\hline
\end{tabular}




\section{LIST OF FIGURES}

1.1 USA frequency allocation chart $[2] \ldots \ldots \ldots \ldots$

1.2 Hong Kong frequency allocation chart [3]. . . . . . . . . . . . 13

1.3 Mauritius frequency allocation chart [4]. . . . . . . . . . . . 14

1.4 Cognitive Radio Concept [5]. . . . . . . . . . . . . . . . . 16

1.5 Haykin's Cognitive Cycle $[6] \ldots \ldots \ldots \ldots \ldots$

1.6 Mitola's Cognitive Cycle $[7] \ldots \ldots \ldots$

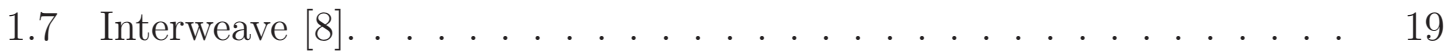

1.8 Underlay $[8] \ldots \ldots \ldots \ldots$

1.9 Overlay $[8] . \ldots \ldots \ldots \ldots$

1.10 Butterfly Network $[9] . \ldots \ldots \ldots$

2.1 Layer prioritization and clique selection For PN and CRN [1]. . . . . 35

2.2 Average recovery overhead vs number of PUs in PN. . . . . . . . . 41

2.3 Average recovery overhead vs number of PPs in PN. . . . . . . . . 41

2.4 Average recovery overhead vs erasure probabilities in PN. . . . . . 42

2.5 Average recovery overhead vs number of SUs in CRN. . . . . . . . 42

2.6 Average recovery overhead vs number of SPs in CRN . . . . . . . . 43

2.7 Average recovery overhead vs erasure probabilities in CRN. . . . . . 43

2.8 Comparing hybrid to no-interweave based systems in PN. . . . . . . 44

2.9 Comparing hybrid to no-interweave based systems in CRN . . . . . . 45

3.1 Average recovery overhead vs number of PUs in PN with FC. . . . . 54

3.2 Average recovery overhead vs number of PPs in PN with FC. . . . . 54

3.3 Average recovery overhead vs erasure probabilities in PN with FC. . 55

3.4 Average recovery overhead vs number of SUs in PN with FC. . . . . 55

3.5 Average recovery overhead vs number of SPs in CRN with FC. . . . 56

3.6 Average recovery overhead vs erasure probabilities in CRN with FC. . 56

3.7 Comparing FC-based and Non-FC based systems in PN. . . . . . 57

3.8 Comparing FC-based and Non-FC based systems in CRN. . . . . . 58 


\section{Chapter 1}

\section{Introduction}

\subsection{Motivation}

The wireless spectrum is becoming more and more licensed with the advancement and penetration of cellular technology. Consumers are quickly reaching hundreds of thousands for new services such as wireless internet access and satellite digital audio broadcasting. This has led to a dramatic growth in the demand for access to spectrum. However, most of the spectrum has already been assigned thus making it more difficult to provide spectrum for new services or for expanding existing ones. For example, the figures below show the frequency allocation in the United States of America (USA), Hong Kong, and Mauritius. The spectrum is almost fully assigned or licensed. Studies performed in USA show that the problem is often a spectrum access problem rather than being a lack of available spectrum problem. These studies have showed that lower frequencies are less utilized than higher frequency bands and that there is a significant amount of "white space" despite the dynamic nature of the land mobile demand. Thus it can be concluded that there exists unexploited capacity in the spectrum [10]. 

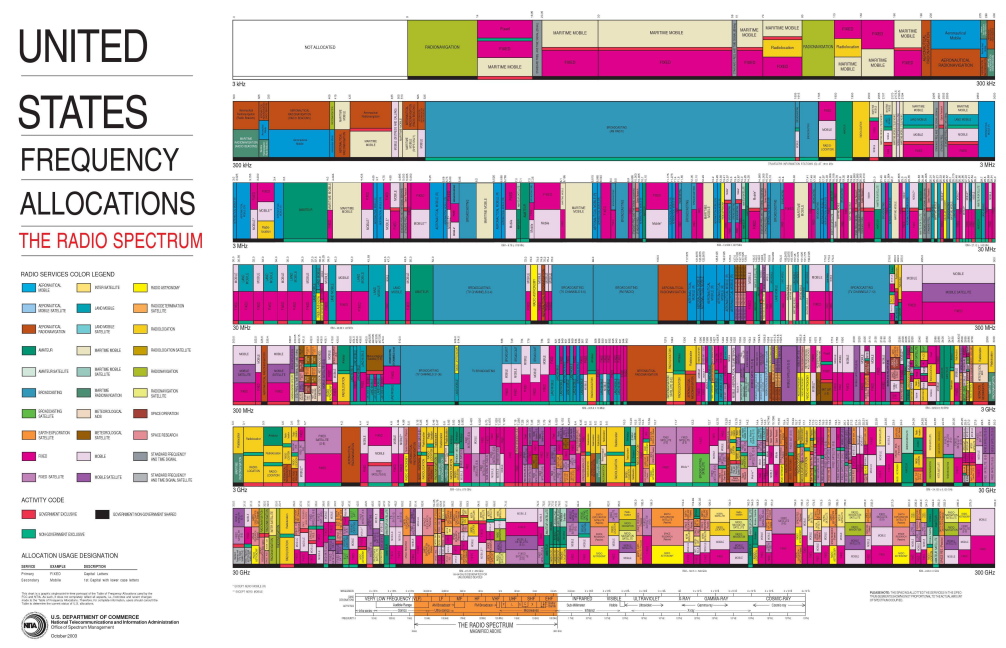

Figure 1.1: USA frequency allocation chart [2].

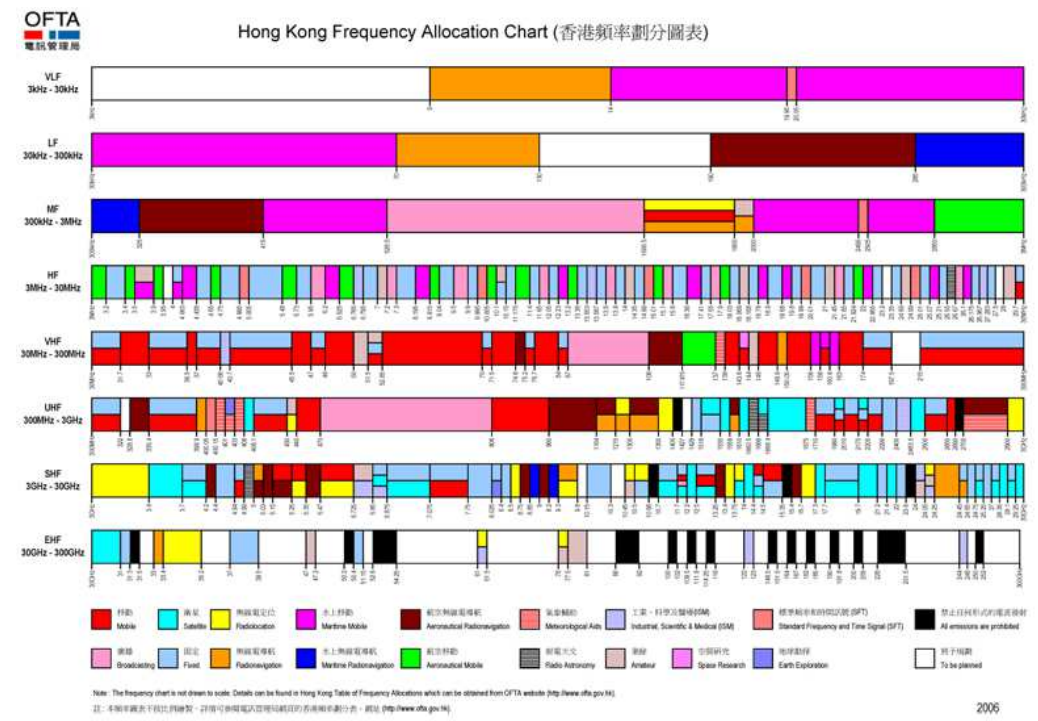

Figure 1.2: Hong Kong frequency allocation chart [3]. 


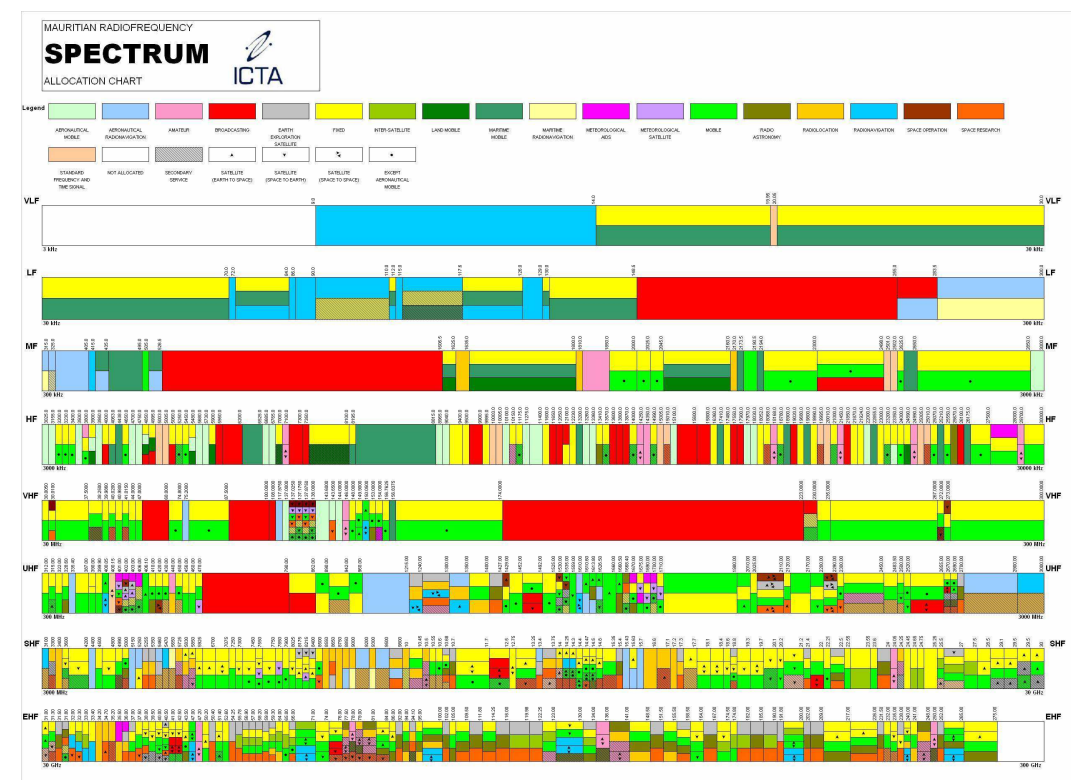

Figure 1.3: Mauritius frequency allocation chart [4].

\subsection{Cognitive Radio Networks}

Cognitive radio (CR) techniques have emerged as a promising solution to utilize the wasted licensed spectrum by allowing the coexistence of primary and secondary networks using dynamic and hierarchical spectrum sharing.

\subsubsection{Definitions}

Different definitions have been given to describe the idea of Cognitive Radio. Different regulatory entities and institutions provide different definitions as can seen below $[5]$ :

- International Telecommunication Union (ITU): A radio or system that senses, and is aware of its operational environment and can dynamically and autonomously adjust its radio operating parameters accordingly.

- Federal Communication Commission (FCC): CR is a radio that can change its transmitter parameters based on interaction with the en- 
vironment in which it operates.

- National Telecommunications \& Information Administration (NTIA):

A radio or system that senses its operational electromagnetic environment and can dynamically and autonomously adjust its radio operating parameters to modify system operation, such as maximize throughput, mitigate interference, facilitate interoperability, and access secondary markets.

- Wireless World Research Forum (WWRF): CR employs a dynamic time-frequency-power based radio measurement and analysis of the RF environment, to make an optimum choice of carrier frequency and channel bandwidth to guide the transceiver in its end-to-end communication, with quality of service being an important design requirement.

The definitions above several common keywords:

- The word "radio"

- Interaction with the environment

- Measuring parameters

- Decision making based on measurements

- Autonomicity

- Adaptation

Figure 1.4 best illustrates the concept of CR.

\subsubsection{History}

The idea originally started by extending the notion of Software Defined Radio (SDR). The need for reconfigurable radio transmitters and receivers that can be 


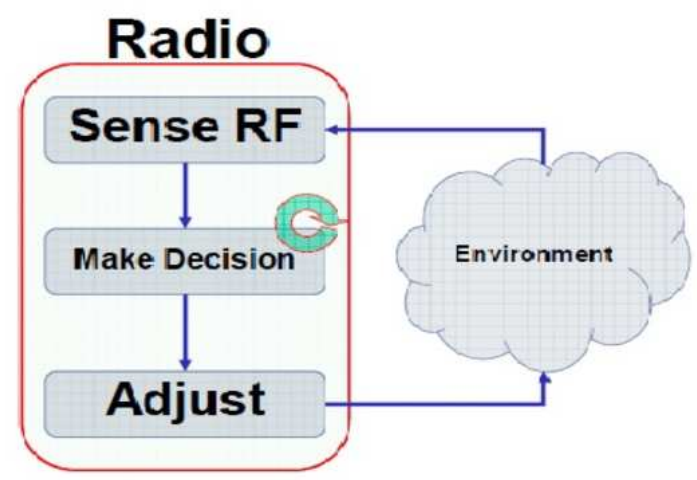

Figure 1.4: Cognitive Radio Concept [5].

updated remotely has prompted the development of SDR [11]. This for example allows remote reconfiguration of the network whenever a change in the traffic pattern occurs. Also, SDR allows the incorporation of several standards or protocols for transmission and reception. This solves the issue of international compatibility since for example the USA use CMDA2000 while GSM is used in Europe [5]. Mitola was the first to come up with the term "Cognitive Radio". In his phD dissertation, Mitola addresses the issue of organizing radio domain knowledge into data structures in real time that integrate machine learning and natural language processing technology into software radio [12]. Mitola wanted to add a "brain" to SDR by trying to join the artificial intelligence world with the radio one while developing XML for radio systems. He extended the notion of SDR to CR by using what is known as "Radio Knowledge Representation Language" (RKRL) to enhance the flexibility of personal services. RKRL allows CR agents to manipulate the protocol so as to service their users in a better way [7]. Mitola's cognitive radio vision can be characterized by [5]:

- User-oriented: Aware of both the radio environment and user environment.

- Multi-Standard: Able to use different communication technologies.

- Terminal-centric: PDA is in itself the CR

- Advanced features: Video processing, voice recognition,... 
Mitola's vision aims to opportunistacally use any available Standard network and improve the user's Quality of Experience (QoE).

Haykin takes a more practical approach to cognitive radio. His vision is much closer to the radio world and focuses mainly on SDR, signal processing, and machinelearning procedures to implement CR. He aims to opportunistically use the spectrum [6]. He highlights three main online cognitive tasks:

- Radio-scene Analysis: which includes detection of spectrum holes and estimation of interference temperature of the radio environment. This is done by the receiver.

- Channel Identification: which includes predicting the channel capacity for the transmitter use as well as estimating the channel-state information (CSI). This is also done by the receiver.

- Transmit-power Control and Dynamic Spectrum Management: This is done by the transmitter.

These tasks form what is known as a cognitive cycle through interaction with the RF environment. Figures 1.5 and 1.6 picture the cognitive cycles of both Haykin's and Mitola.

\subsubsection{Techniques}

There are three main techniques in CR [8]:

- Interweave: In this technique, the CR detect a spectrum hole and utilize it. The CBS only transmits whenever the PBS is silent. This ensures that there is no interference to the PUs caused by the CBS.

- Underlay: In this technique, the CBS constantly transmits, but with a low power so as the interference it causes by its transmission is below a defined 


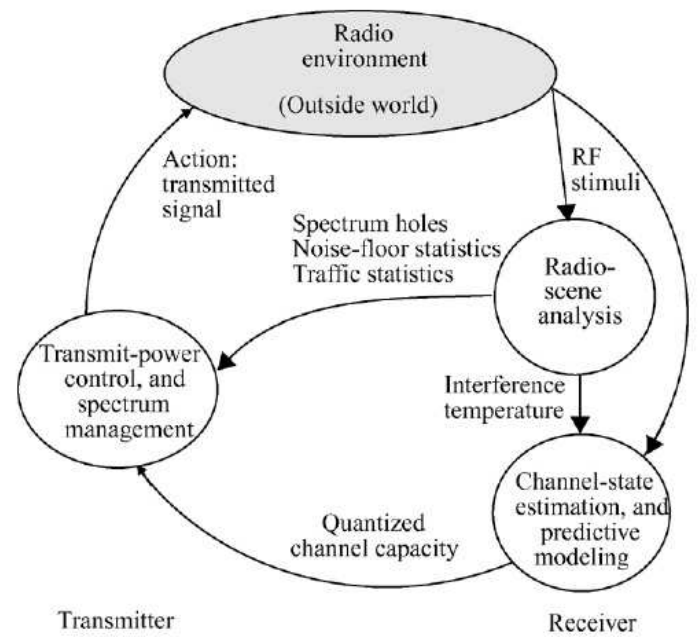

Figure 1.5: Haykin's Cognitive Cycle [6].

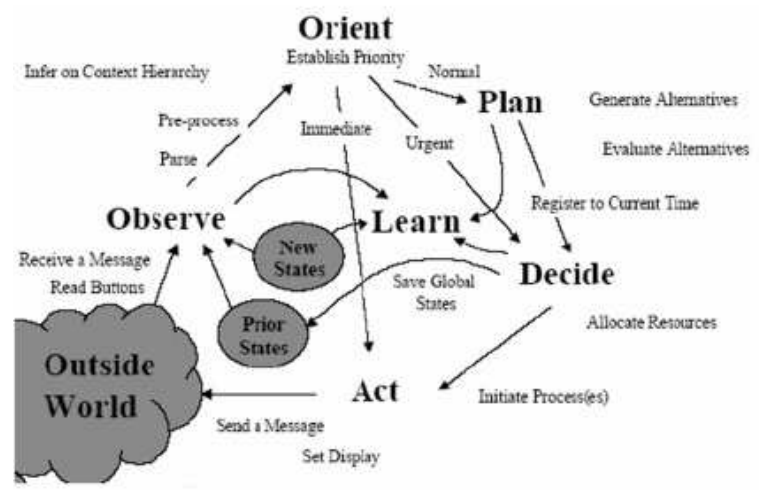

Figure 1.6: Mitola's Cognitive Cycle [7]. 
threshold. This ensures that the interference towards the PUs is controlled rather than avoided.

- Overlay: In this technique, the CBS also constantly transmits with low power to control the interference. However, the CBS has some knowledge of the codebooks used and the messages sent by the PBS and thus it can help the PBS in cases of message retransmission.

Interweave and underlay are more prevalent as they offer more security to the PUs since the CBS has no knowledge of what is being sent or the codebooks used. These three techniques are illustrated in figures 1.7, 1.8, and 1.9
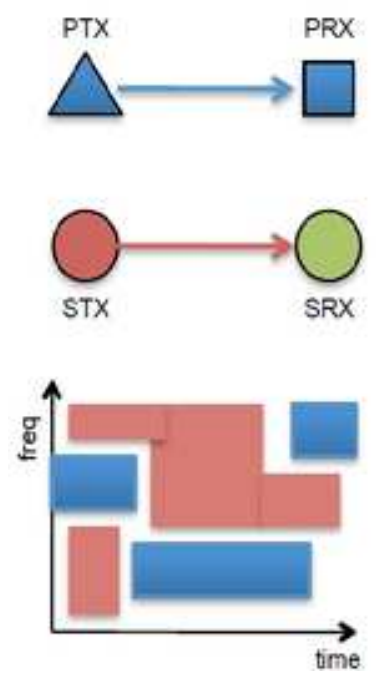

Figure 1.7: Interweave [8].

\subsubsection{Applications}

Due to recent advancements in the software and hardware fields, CR has become a more possible technology to implement. Several applications that can utilize CR technology have been suggested $[13,14]$ :

- Smart Grid Networks: Governments are employing smart power grids in 

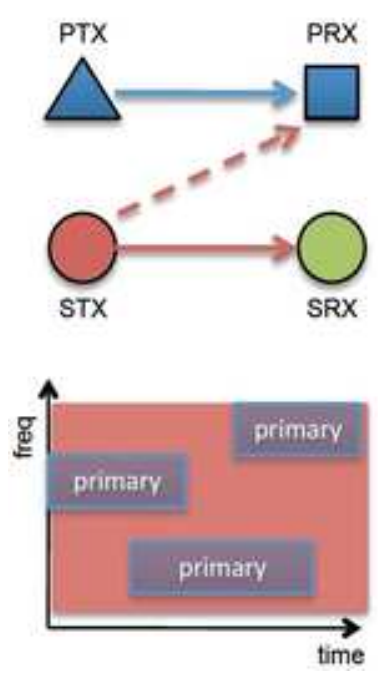

Figure 1.8: Underlay [8].
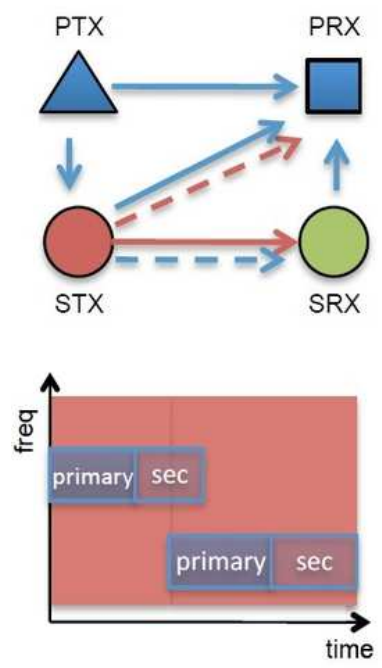

Figure 1.9: Overlay [8]. 
which consumers and households can either consume or produce power depending on their needs at any particular time instance to improve the efficiency of the power production process and achieve energy independence and sustainability. This move from a traditional power grid to a smart power grid has encouraged governments to look for some intelligence to employ in these smart grids. CR-based communication network used to control the grid can offer advantages in terms of bandwidth, distance and cost when compared to current wireline/wireless technologies. Network gateways and smart meters utilize CR and dynamically use underutilized spectrum in order to communicate with each other or with the main power production plant to decide how they should operate at a specific moment depending on the state of the power grid.

- Public Safety Networks: Due to the high congestion of the radio frequencies assigned for public safety use, CR can address this issue by allowing public safety officers to use other frequency bands that are less congested. This is especially beneficial in cases of emergency such as floods where there is a critical need for fast response and ability to communicate information as fast as possible.

- Cellular Networks: With the growth of the cellular networks, customers have become more demanding as they expect to be connected anytime and anywhere. Moreover, the increased popularity of social networks and the introduction of smart phones have dramatically increased the demand for data services. This has put more pressure on cellular operators. Thus operators are looking for CR technologies to help meet this high demand for data by allowing the spectrum to be sensed and shared when possible.

- Wireless Medical Networks: The need to eliminate the need for wired vital signs monitoring of patients has led to the emergence of medical body area networks (MBANs). These MBANs allow the constant monitoring of patients 
continuously and offer the ability to detect any changes at an early stage so that adequate actions can be taken. They also reduce the possibility of infection. However, these MBANs need a relatively clean and less crowded spectrum band for reliable transmission of the patients' information. The industrial, scientific, and medical (ISM) band is not adequate for such applications. Thus CR technology can offer a solution and alternative as it will allow the MBANs to transmit the data in a more reliable manner.

- Military: CR technology offers protection of communication transmission, discovery of paths of opportunity and recognition of enemy communications. This is due to the frequency flexibility and interoperability that CR technologies offer. Thus CR technologies are extremely important for military applications.

\subsubsection{Challenges}

CR faces several challenges for it to be implemented [15]:

- The hardware requirements and the network architectures needed will surely pose another challenge for CR technology since an intelligent and reliable coordination mechanism that allows the coexistence and cooperation within the network is needed.

- Cognitive control channel is essential. Thus a cooperative multi-radio system with terminal devices equipped with SDR capability is necessary for CRN.

- Routing and network security are a basic challenge since the duration of available link is usually only a fraction of inter arrival time.

- $\mathrm{CRN}$ has to be able to deal with different RF spectrum and baseband varieties concurrently since the nodes in a CRN belong to different networks and 
thus might operate using different parameters with different frequency bands at different locations.

- New algorithms and protocols for spectrum sharing are needed so that mobile terminals can communicate with different systems that utilize different algorithms to those implemented for baseband processing and channel coding.

- The high power consumption of CRN nodes is a prevalent challenge that needs to be addressed. This is due to the fact that the CRN node is required to sense a larger frequency band in order to detect any spectrum hole. Furthermore, the CRN node is also required to perform computationally demanding signal processing tasks with relatively low delay which increases its power consumption, especially if a dual-radio architecture is employed for spectrum sensing [16].

- The development of spectrum sensing techniques that are able to detect weak PU signals. This will require the $\mathrm{CRN}$ to have a large amount of CSI to ensure efficient discovery and cooperation techniques. In addition to that, spectrum sensing techniques should be able to deal with issues such as channel uncertainty, noise uncertainty, and aggregate-interference uncertainty [17].

\subsection{Network Coding}

Network coding is a relatively young concept. The seminal paper [18] written by Ahswede, Cai, Li and Yeung is considered to the "birthplace" of network coding. Thus there is slight confusion mixed with excitement about its possibilities and skepticism about its benefit and potential. The concept of network coding was suggested as an alternative to routing [19]. This was due to the long delays that usually occurred when using a traditional packet-switched network model. When using network coding, devices called coders replace the routers and switches in a network. These 
coders transmit metadata along multiple paths simultaneously. This helps reduce the severity of bottlenecks on links and thus reduces the delays for packet arrival.

\subsubsection{Definitions}

Network coding can not be defined in a straight forward manner as there are different definitions used [9]:

- Network coding is the coding at a node in a packet network with error-free links [18].

- Network coding is a technique to optimize the flow of digital data through the transmission of digital evidence about messages [20]. When bits of digital evidence reach the destination, the transmitted message can be deduced rather than reconstructed.

\subsubsection{Types}

There are different types of network coding schemes available to be implemented depending on the application in which they are needed. These types include: linear codes, nonlinear codes, static codes, and convolutional codes [9]. However, in our work in this thesis, we focus on one class of codes which is the linear codes.

We focus particularly on two different network coding schemes:

- Instantly Decodable Network Coding (IDNC): A subsection of the linear network coding schemes that allows instantaneous decoding of the transmitted coded packet [21]. The encoding occurs by simply using binary XOR for the packets to be transmitted and the decoding occurs by applying another binary XOR to eliminate known packets [22]. The condition for instantaneous decodability is that receivers should only be missing one packet of the combined packets it receives. Moreover, it also needs more frequent feedback and more 
complex decision making for packet encoding. However, it was shown that this scheme achieves better decoding delay (time needed to receive and decode all wanted packets) performance than other schemes [22].

- Random Network Coding (RNC): A subsection of the linear network coding schemes that randomly chooses a set of packets to linearly combine with random coefficients [9]. It is shown that RNC is throughput optimal. However, it does increase the decoding delay since the receivers have to wait to receive several coded packets in order to be able to decode the packets [23] .

\subsubsection{Benefits}

Network coding can have profound benefits when utilized in a network. It can improve:

- Throughput: Network coding increases the throughput through the efficient use of packet transmission since we are communicating more information with few packet transmissions. This is best illustrated in the example given by Ahlswede et al [18] which is known as the butterfly network. In this network, two packets $b_{1}$ and $b_{2}$ are present at the source node $s$ and we wish to transmit them both to the destination nodes $t_{1}$ and $t_{2}$.

- Robustness: Network coding offers robustness against two issues: packet losses and link failures. Random linear network coding for example helps address the issue of packet losses. Moreover, network coding can improve resource usage by allowing the network resources to be shared among different paths, thus addressing the issue of link failure.

- Complexity: Network coding helps reduce the complexity of some systems. For example, in the case of minimum-cost subgraph selection for multicast, 


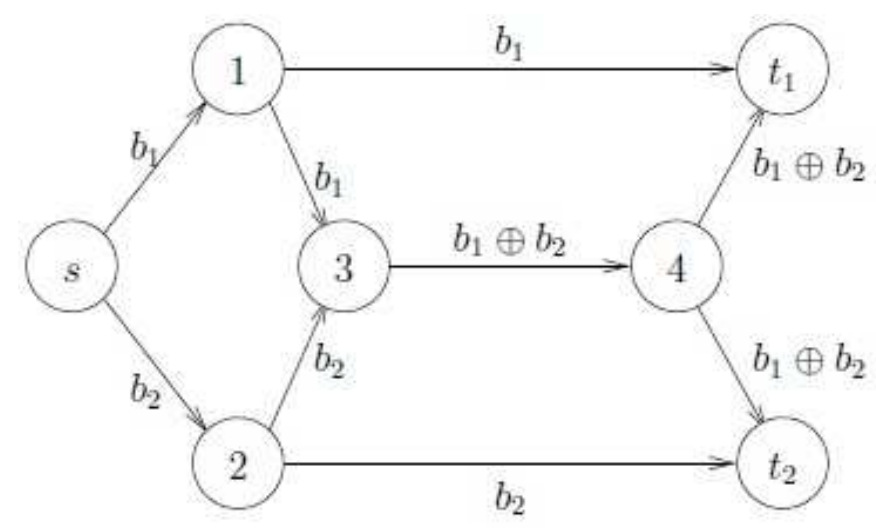

Figure 1.10: Butterfly Network [9].

routing involves Steiner trees which is by itself a complex process. However, by using network coding, the problem becomes a linear optimization problem that has a low-complexity distributed solution.

- Security: Network coding can offer a security benefit and be used as a possible mechanism for secure communication. For example, using the same butterfly network model, if a malicious node only obtains $b_{1} \oplus b_{2}$, it will not be able to obtain either $b_{1}$ or $b_{2}$, thus creating a secure link.

\subsubsection{Applications}

The improvement in network throughput offered by the use of network coding is dependent on the topology of the network as well as the frequency and severity of bottlenecks. It can never reduce the throughput when compared to conventional routing. Thus, network coding can be particularly helpful in :

- Multicast Networks: The use of network coding can be very beneficial in multicast networks. Work done in [24] shows that if sources are encoded optimally, network coding can reach the optimal bound of the admissible coding rate. 
- Wireless Sensor Networks: Network coding can also increase the information content of transmissions in wireless sensor networks. In paper [25], tests showed that the throughput of a 20-node wireless network increased and that the gains varied from a few percent to several folds depending on the transport protocol, level of congestion, and the patter of the traffic.

- Peer-to-Peer File Sharing (P2P): Network coding can also be used in P2P networks. The arbitrary topology nature of the P2P network and the fact that nodes in a P2P network are end hosts that are capable of performing complex operations make it perfect to use network coding in such networks. Simulations performed in [26] show an increase of $15 \%$ - $20 \%$ in the network throughput.

\subsection{Thesis Organization}

The remainder of this thesis is organized as follows:

Chapter 2 describes the system model employed in this thesis and discusses the performance gain of using the proposed collaborative instantly decodable network coding (IDNC) based scheme on a hybrid cellular cognitive radio network.

Chapter 3 studies the performance of the same network when employing fractional cooperation (FC) as a spectrum shaping mechanism for the primary network traffic. Chapter 4 summarizes the results obtained in this thesis and discusses possible future works. 


\section{Chapter 2}

\section{Collaborative Multi-Layer Network Coding For Hybrid Cellular Cognitive Radio Networks}

\subsection{Previous Work}

To be able to implement the two main techniques flexibly in cognitive radio networks (CRN), several works have suggested dynamic spectrum allocation solutions. These works have been extended in [27] to the multi-cell case by presenting a structure for cellular primary and cognitive radio multi-cell coexistence with limited coordination, using intra-cell spectrum interweave and inter-cell spectrum underlay.

Network coding has been suggested as a possible PN traffic spectrum shaping technique to increase opportunities for the CRN and thus improving its performance [28-30]. It has shown that it can significantly improve transmission efficiency, throughput and delay over wireless erasure channels [22,31-42]. It can provide several more spectrum holes for the CRN to use by speeding up the packet transmission and recovery in the PN. In [1], spectrum underlay and network coding were combined to improve the performance, quantified by the average recovery overhead (defined to 
be the percentage ration of the average completion delay to the frame size), of both networks without jeopardizing the performance of each. The improvement in the average recovery overhead indicated that there is also an improvement in the average received throughput in each network since a lower average recovery overhead implies that we can transmit new information within a specific time duration. However, it only considered the case of high primary traffic loads, which is not general since cases of low loads can occur.

In this chapter, we extend the work done in [1] by utilizing the same multi-layer network coding scheme when both spectrum interweave and underlay are allowed to be used opportunistically by the secondary network. This allows us to study the performance, quantified by the average recovery overhead, of the suggested scheme in a more general context in which we operate adaptively in both low and high primary traffic loads cases. The overhearing capability of both base stations is exploited to speed up the packet recovery process of both networks using instantly decodable network coding (IDNC). The same prioritized multi-layer IDNC technique is used to guarantee that this collaboration does not degrade the performance of each network. On the contrary, when this scheme is utilized in a hybrid CRN (characterized by the two new phases that represent the higher power transmission of new packets as well as recovering lost ones by the CRN base station when the PN is silent), we observe significant improvement in performance due to both the collaborative packet recovery scheme as well as the ability to utilize the available primary network spectrum holes.

To calculate the average recovery overhead:

$$
\text { Average Recovery Overhead }=\frac{\text { Average Completion Delay }}{\text { Frame Size }} * 100
$$

where the average completion delay is the number of transmissions sent by the basestation to recover lost packets of a given frame. 


\subsection{System Model}

The model considered in this thesis consists of multi-cell PN and multi-cell CRN in such a way that each PN cell and CRN cell are collocated. For each network, a cell contains a base station and a set of users, i.e a PN cell consists of a primary base station (PBS) and $M_{P}$ users (PUs) while a CRN cell contains a cognitive radio base station (CBS) and $M_{S}$ secondary users (SUs). A subchannel reuse factor of less than 1 is used by both networks, implying that different subchannels are used by adjacent cells.

Each collocated PBS and CBS use different subchannels whenever there is no spectrum hole in the PN (i.e. as long as PN has packets to transmit or recover). In this case, the CBS will employ a subchannel of an adjacent cell using spectrum underlay. This implies that the PUs have to tune their receivers to the subchannels of adjacent cells in addition to those used in the cell in order to benefit from the transmission of the CBS. It is assumed that the CBS utilizes any of the discussed power optimization schemes in spectrum underlay literature in order to ensure the interference threshold condition at the PUs is met. However, whenever a spectrum hole is detected, the CRN switches to the PN subchannel to transmit at higher power.

Another assumption made is perfect sensing which allows the CBS to exactly detect the presence of a spectrum hole. The CBS constantly senses whether the PBS is transmitting or not and perfectly detects the start spectrum holes.

A unicast or multicast scenario is assumed on both networks. The PBS broadcasts frames of $N_{P}$ packets (PPs) while the collocated CBS broadcasts frames of $N_{S}$ packets (SPs). The users of each network are interested in receiving distinct or partially overlapping subsets of the broadcasted packets from their respective base stations. However, due to the broadcast nature of the wireless channel, both the PBS and CBS

as well as the PUs and SUs can overhear each other's packets on both the PBS and CBS frequencies and temporarily store these overheard packets for possible future 
use.

In this thesis, we adopt a MAC layer view of all channel impairments in the form of packet erasures.. The packet erasure probabilities for any user $i$ are denoted by $p_{i}^{(p)}$ and $p_{i}^{(s)}$ on the primary and secondary subchannels respectively. It is clear that $p_{i}^{(s)}$ is higher than $p_{i}^{(p)}$ since the CBS is transmitting at a lower power than the PBS and its transmission is subjected to higher levels of interference from adjacent cell PBSs.. However, when PBS is silent, the CBS transmits at a higher power while having no

interference from the PBS. Thus the erasure probability for the CBS will become $p_{i}^{(p)}$ whenever the PBS is silent. An acknowledgement is sent on the signalling channel and is overheard by both base stations whenever a packet is received on any of the subchannels.

Note that frame synchronization is not required between the two networks. Moreover, no additional coordination or exchange of information is needed on top of what is required in [27].

\subsection{Multi-Layer IDNC Graph and Algorithm}

\subsubsection{Graph Theory Basics}

It is assumed that each base station transmits a set of $\mathrm{N}$ source packets to a set of users in a unicast or multicast scenario. When the initial transmission phase ends in which the $\mathrm{N}$ packets are transmitted uncoded, three possible feedback sets can be associated with each user $i$ :

- The Has set: The set of packets correctly received by $U_{i}\left(\right.$ Denoted by $\left.H_{i}\right)$.

- The Lacks set: The set of packets not received by user $U_{i}$ (Denoted by $L_{i}$ ).

- The Wants set: The set of requested packets not received by user $U_{i}$ (Denoted by $\left.W_{i} \subseteq L_{i}\right)$. 
The IDNC graph allows us to determine the packets that can be combined and recovered simultaneously in the same transmission rather than having to recover the packets in the Wants set of each user by sending them individually. To construct the graph $G$, a vertex $v_{i j}$ is generated in $G$ for each packet $j \in L_{i}$. Two vertices $v_{i j}$ and $v_{k l}$ are said to be adjacent in $G$ if one of the below conditions is satisfied:

- Condition 1: $j=l \Rightarrow$ Two users $i$ and $k$ lost the same packet $j$.

- Condition 2: $j \in H_{k}$ and $l \in H_{i} \Rightarrow$ The lost packet of user $i$ is in the Has set of user $k$ and vice versa.

Therefore, each edge connecting two vertices $v_{i j}$ and $v_{k l}$ in $G$ implies that the lost packets $j$ and $l$ at users $i$ and $k$ can be instantly and simultaneously served by either transmitting packet $j$ if $j=l$ or the coded packet $j \oplus l$ if $j \in H_{k}$ and $l \in H_{i}$. For each network, we divide the vertices into two groups:

- Target Subgraph: It contains all the vertices from the Wants set of all users.

- Auxiliary Subgraph: It contains all the vertices in the Lacks set but not in the Wants set of any user.

\subsubsection{Clique Selection Algorithm}

To be able to serve the aforementioned vertices, we need to find the largest number of connected vertices in these subgraphs. This is known to be the Maximal Clique Problem $(M C P)$. A clique is defined to be a subset of the graph whose vertices are all adjacent/connected to one another. Therefore, each clique represents a packet combination that can instantly serve all the users generating the vertices of the clique [33]. The MCP is a problem that aims to find the largest clique in a given graph. It is an NP-hard problem [43]. Therefore, we usually apply heuristic algorithms to find this maximal clique. 
Any clique selection algorithm chosen to optimize a specific parameter is applied firstly to the target subgraph as it represents the actual set of missing packets that need to be served and thus must be the main focus of the algorithm. Once the optimal clique $K^{*}$ is found in the target subgraph, the subset of users to be served with one of their lost packets and the corresponding packet combination are determined. After that, the fully connected auxiliary to optimal clique $K^{*}$, which is the set of vertices in the auxiliary subgraph that are each adjacent to all the vertices $\in K^{*}$, is extracted and the same clique selection algorithm is run on it to determine the maximal clique possible. This allows us to find new possible packets to combine with the previously determined ones to serve other users with auxiliary packets without violating the instant decodability condition at the users determined by $K^{*}$.

\subsubsection{Multi-layer IDNC Algorithm}

The same concept can be extended to a multi-layer graph which is composed of four layers, a target subgraph and an auxiliary subgraph for each of the two networks. IDNC can be used by each network by giving higher priority for recovering its own packets and lower priority to helping the other network recover its packets. The same conditions apply to determine the adjacency between vertices. However, these vertices are classified in different layers, and subsequently different priorities. To find the optimal clique, each network applies the clique selection algorithm to the higher priority layers first (i.e its own target and auxiliary layers) to guarantee identifying the same set of packets that would have been sent if there was no collaboration. Whenever this clique is identified, the algorithm now moves to lower priority layers to identify all the packets of the other network that can be simultaneously served with its own packets without violating the instant decodability property. This ensures that each network guarantees that its own performance does not deteriorate while helping the other network whenever it is possible. 
Throughout our work, we use the maximum weight clique selection algorithm. It is shown in [32] that such a clique selection algorithm reduces the recovery overhead. This clique selection algorithm is dependent on two main factors: the size of the Wants set of each user as well as its erasure probability. Thus, to reduce the recovery overhead, we give higher weights to vertices with larger Wants set and higher erasure probability. Therefore, we define for each vertex the individual mean completion time $\tau_{i}^{j}(z)$ (the expected time for receiver $\mathrm{i}$ at state $\mathrm{z}$ to receive all its missing packets if addressed in all future transmissions) to be: $\tau_{i}^{p}(z)=\left(\frac{\left|W_{i}\right|}{1-p_{i}^{(p)}}\right)^{n}$ for PN vertices and $\tau_{i}^{S}(z)=\left(\frac{\left|W_{i}\right|}{1-p_{i}^{(s)}}\right)^{n}$ for CRN vertices where $n$ is a biasing component. We apply a simple greedy vertex heuristic in which we search for the maximum weight vertex which is a simpler algorithm to implement [33]. This is done by firstly defining the adjacency indicator $a_{i j, k l}(z)$ between vertices $v_{i j}$ and $v_{k l}$ in the graph $G(z)$ such that:

$$
a_{i j, k l}(z)= \begin{cases}1, & \text { if } v_{i j} \text { is adjacent to } v_{k l} \text { in } G(z) \\ 0, & \text { otherwise. }\end{cases}
$$

Then we define the weighted degree $\Delta_{i j}(z)$ of vertex $v_{i j}$ as:

$$
\Delta_{i j}(z)=\sum_{\forall v_{k l} \in G(z)} a_{i j, k l}(z) \tau_{k}^{j}(z)
$$

A large weighted vertex degree reveals its connection to a large number of vertices belonging to receivers with large values of $\tau_{i}^{j}(z)$. Now the vertex weight $w_{i j}(z)$ can be defined as:

$$
w_{i j}(z)=\tau_{i}^{j}(z) \Delta_{i j}(z)
$$

Thus to find the maximum weight clique, we start by finding the maximum weight vertex and set it to be the source node. We then search for the next maximum weight vertex connected to this source node and so on. As mentioned before, the search 
starts in the target subgraph of the intended network, then moves on to its auxiliary subgraph, then to the target subgraph of the other network, and finally the auxiliary subgraph of the other network. This is shown in figure 2.1.

\begin{tabular}{c|c|c|c|}
\multicolumn{1}{c}{} & \multicolumn{1}{c}{ PP 1 } & \multicolumn{1}{c}{ PP 2 } & \multicolumn{1}{c}{ PP 3 } \\
\cline { 2 - 4 } PU $1_{\text {P }}$ & 1 & -1 & 0 \\
\cline { 2 - 4 } PU 2 & 0 & 1 & 1 \\
\cline { 2 - 4 } & &
\end{tabular}

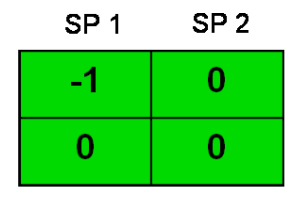

\begin{tabular}{|c|c|c|c|}
\cline { 2 - 3 } su 1 & 0 & -1 & 0 \\
\cline { 2 - 4 } su 2 & -1 & 0 & 0 \\
\cline { 2 - 4 } & &
\end{tabular}
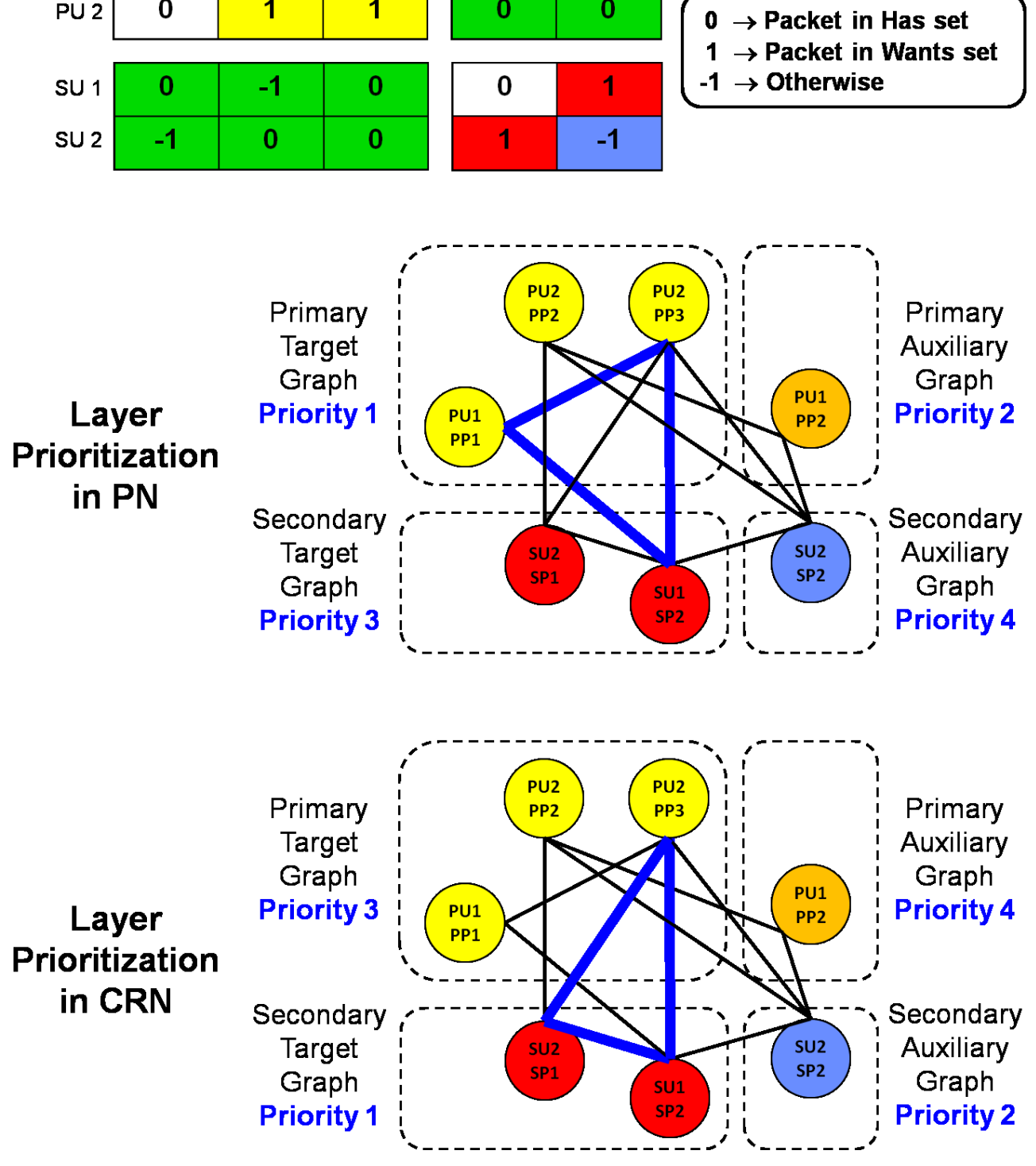

Figure 2.1: Layer prioritization and clique selection For PN and CRN [1]. 


\subsection{Proposed IDNC-based Packet Recovery Algo- rithm}

The same Multi-Layer IDNC graph and algorithm used in [1] is used here. The proposed algorithm runs on six different phases based on the state of the two networks:

\section{$\circ$ Phase 0: PN sends - CRN sends:}

Both the PN and the CRN send new frames of uncoded source packets in their initial transmission phase. No collaboration is needed in this phase. However, the PBS and CBS as well as all the PUs and SUs overhear each other's packets to use them in later phases. Due to the fact that the networks do not need to be synchronized, this phase can move to one of three phases as follows:

- Both networks finish sending the new frames simultaneously $\Rightarrow$ Phase 1.

- Only PN finishes sending its new frame $\Rightarrow$ Phase 3 .

- Only CRN finishes sending its new frame $\Rightarrow$ Phase 2 .

\section{○ Phase 1: PN recovers - CRN recovers:}

Both networks recover the lost packets of its users. The multilayer IDNC graph is built and prioritized as shown in the example shown in Figure 2.1 by each of the base-stations. They then generate their coded packets independently based on their priorities and send them over their respective subchannels. The collaboration in recovery can aid both networks in decreasing their recovery overhead. The graph is updated when the feedback is received. This procedure is repeated until one of three events occurs:

- Both networks finish recovery simultaneously:

- If there is a new arrival on $\mathrm{PN} \Rightarrow$ Phase 0 . 
- If there is no new arrival on PN $\Rightarrow$ Phase 4.

- Only PN finishes packet recovery:

- If there is a new arrival on $\mathrm{PN} \Rightarrow$ Phase 2.

- If there is no new arrival on PN $\Rightarrow$ Phase 5.

- Only CRN finishes packet recovery $\Rightarrow$ Phase 3 .

$\circ$ Phase 2: PN sends - CRN recovers:

A new frame of packets is sent uncoded on the primary subchannel by the PBS which does not help the CRN. In the mean time, the CRN uses the multilayer IDNC algorithm to recover its packets as well as those of the PN. This permits the PUs to receive new packets on the primary subchannel while simultaneously recovering their lost packets on the secondary subchannel. This will significantly reduce the PN's recovery phase and overhead. This is repeated until one of the following three events occurs:

- PN finishes sending its new frame while CRN simultaneously finishes packet recovery $\Rightarrow$ Phase 3 .

- PN finishes sending its new frame while $\mathrm{CRN}$ is still recovering $\Rightarrow$ Phase 1.

- CRN finishes packet recovery while PN is still sending its new frame $\Rightarrow$ Phase 0.

\section{○ Phase 3: PN recovers - CRN sends:}

This is the complete opposite of phase 2. During this phase, a new frame of packets is sent by the CBS, which does not help the PN, over the secondary subchannel. But the CBS as well as the SUs still buffer the overheard packets that the PN is still trying to recover. In the mean time, the PN uses the multilayer IDNC algorithm to recover its packets on the primary subchannel as well as simultaneously hearing the new 
packets of the CRN. The PBS updates the overhearing information of the packet in its prioritized multi-layer IDNC graph after each new packet transmission. This permits the SUs to receive new packets on the secondary subchannel while simultaneously recovering their lost packets on the primary subchannel. This will significantly reduce the CRN's recovery phase and overhead. This procedure is repeated until one of three events occurs:

- CRN finishes sending its new frame while PN simultaneously finishes packet recovery

- If there is a new arrival on $\mathrm{PN} \Rightarrow$ Phase 2 .

- If there is no new arrival on $\mathrm{PN} \Rightarrow$ Phase 5.

- PN finishes packet recovery while CRN is still sending its new frame

- If there is a new arrival on $\mathrm{PN} \Rightarrow$ Phase 0 .

- If there is no new arrival on $\mathrm{PN} \Rightarrow$ Phase 4.

- $\mathrm{CRN}$ finishes sending its new frame while $\mathrm{PN}$ is still recovering $\Rightarrow$ Phase 1.

$\circ$ Phase 4: PN silent - CRN sends:

In this phase, the CBS takes advantage of the fact that there are no packets being sent to the PUs by the PBS. This allows it to transmit on the primary channel with a higher power without fear of causing interference at the PUs. This helps in decreasing recovery overhead as transmitting with higher power results in a lower error probability. The CBS is constantly sensing whether there is a new batch of packets that will be transmitted by the PBS. The system can move from this phase to any of the following three phases depending on the following events:

- CRN finishes sending its new frame and PN simultaneously has a new arrival $\Rightarrow$ Phase 2 . 
- $\mathrm{CRN}$ finishes sending its new frame and PN has no new arrival $\Rightarrow$ Phase 5.

- $\mathrm{CRN}$ is still sending its new frame and PN simultaneously has a new arrival $\Rightarrow$ Phase 0.

\section{○ Phase 5: PN silent - CRN recovers:}

This phase is very similar to Phase 4 in that the CBS sends on the primary subchannel with high power and less erasures due to the silence of the PBS. The only difference is that the CRN is recovering its packets and not sending new uncoded packets as in Phase 4. Again, depending on the following events, the system can move to one of the following three phases:

- $\mathrm{CRN}$ finishes packet recovery and PN simultaneously has a new arrival $\Rightarrow$ Phase 0.

- $\mathrm{CRN}$ finishes packet recovery and PN has no new arrival $\Rightarrow$ Phase 4 .

- $\mathrm{CRN}$ is still recovering packets and PN simultaneously has a new arrival $\Rightarrow$ Phase 2.

\subsection{Simulation Results}

A comparison is performed through simulation between four different schemes:

- Proposed Collaborative scheme (C-IDNC): IDNC based scheme in which a clique is selected from all the subgraphs based on the priorities set for each subgraph.

- Non-Collaborative scheme (NC-IDNC): IDNC based scheme in which a clique is selected only from the target subgraph of each network. 
- Random network coding based scheme (RNC): RNC based scheme in which a random set of packets from the vertices found in the target subgraph of each network is selected and combined for transmission.

- Automatic Repeat Request based scheme (ARQ): The basic ARQ scheme in which the base station retransmits each packet in the Wants set of each user one by one until a positive acknowledgement that all these packets are correctly received and decoded arrives at the base station. Users do not store partial data received from any retransmission. They either send a positive or negative acknowledgement.

When plotting against any parameter, the remaining parameters are set as follows: number of PUS and SUs is set to 50, number of PPs and SPs is set to 30, and the erasure probabilities of primary and secondary subchannel varies in the interval $\left[\begin{array}{ll}0.01 & 0.2\end{array}\right]$ and $\left[\begin{array}{ll}0.21 & 0.4\end{array}\right]$ respectively. The average recovery overhead, which is the percentage ration of the number of transmissions needed to recover lost packets of a given frame to the frame size, is plotted against any varying parameter.

Note that:

- Solid lines are used to characterize the performance of the PN while dashed lines are used to characterize the performance of the CRN.

- Curve fluctuations are due to finite simulation runs.

Figures 2.2, 2.3 and 2.4 show that for the PN, the proposed collaborative scheme outperforms the non-collaborative scheme by around $12 \%$, the RNC scheme by around $24 \%$ and the ARQ scheme by $30-90 \%$ (depending on the metric). This is in accordance with previous results obtained in [1].

Figure 2.5, 2.6 and 2.7 shows that for the CRN and for a primary packet probability of arrival of 0.1 , the collaborative scheme outperforms the non-collaborative 


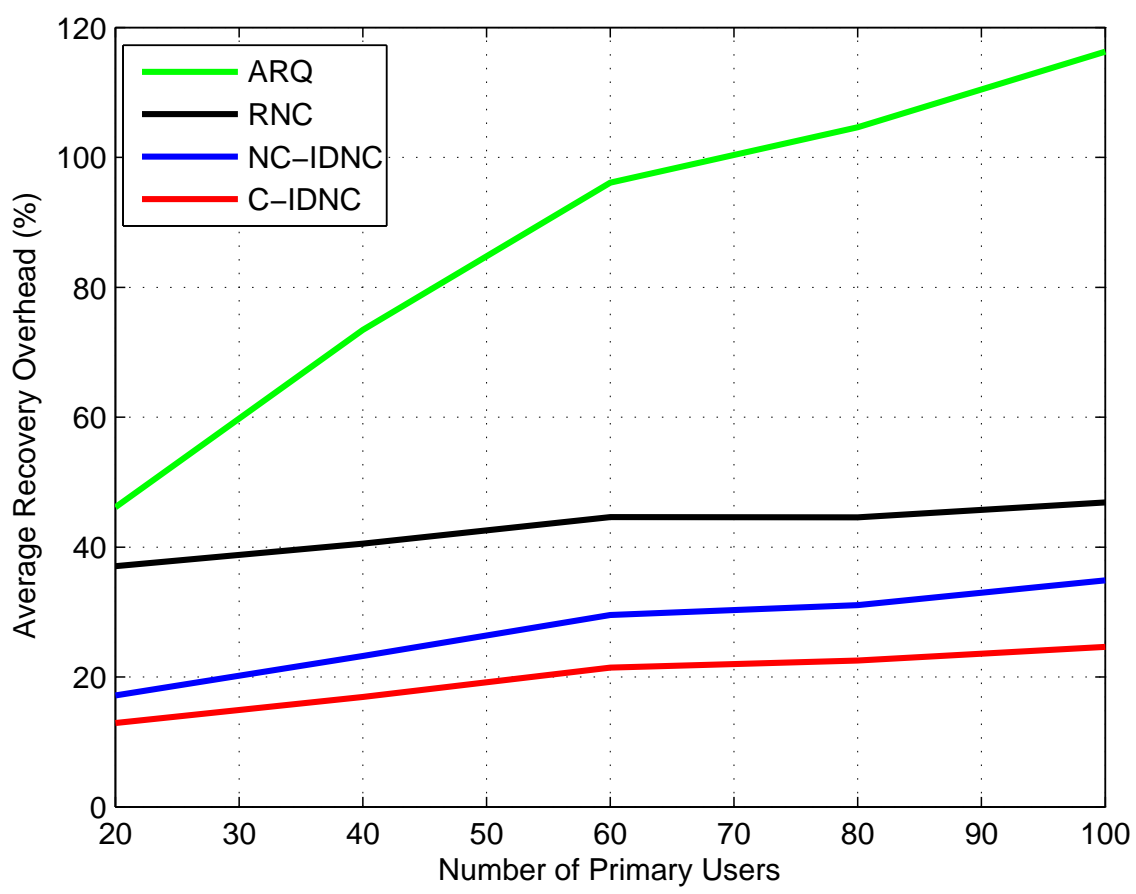

Figure 2.2: Average recovery overhead vs number of PUs in PN.

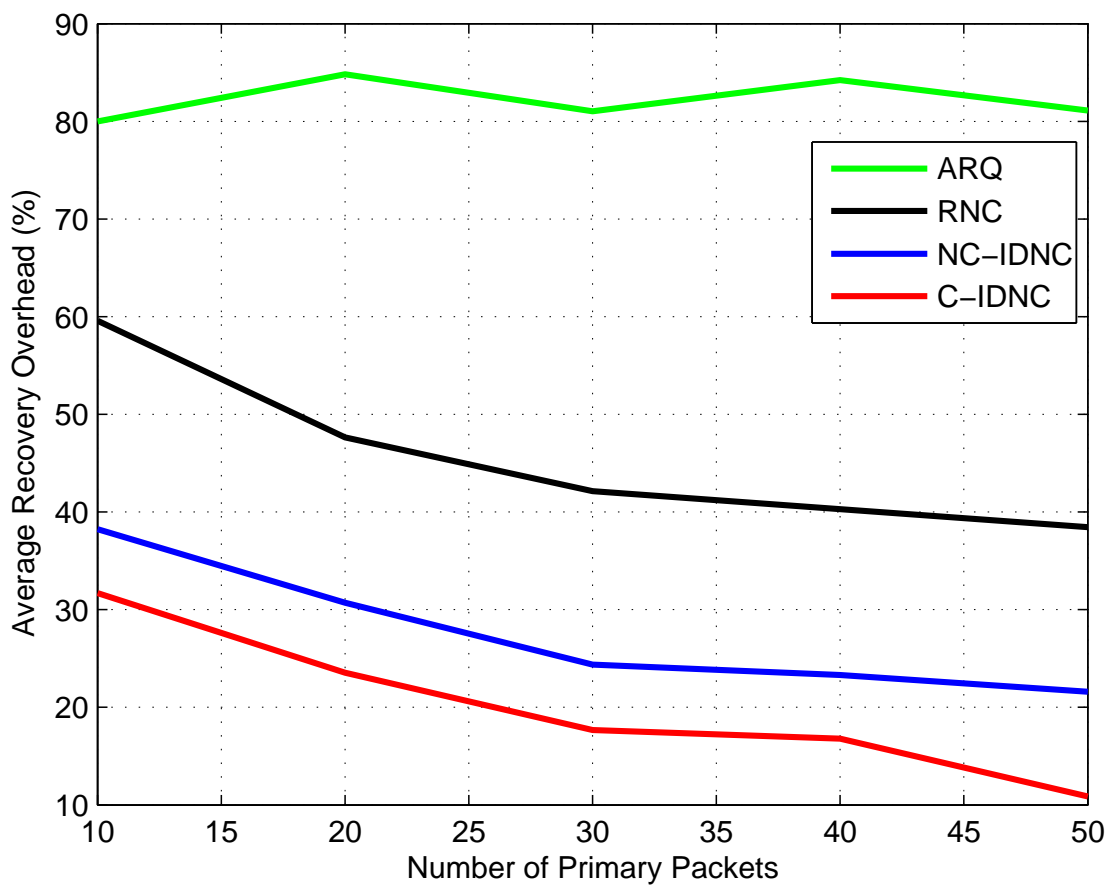

Figure 2.3: Average recovery overhead vs number of PPs in PN. 


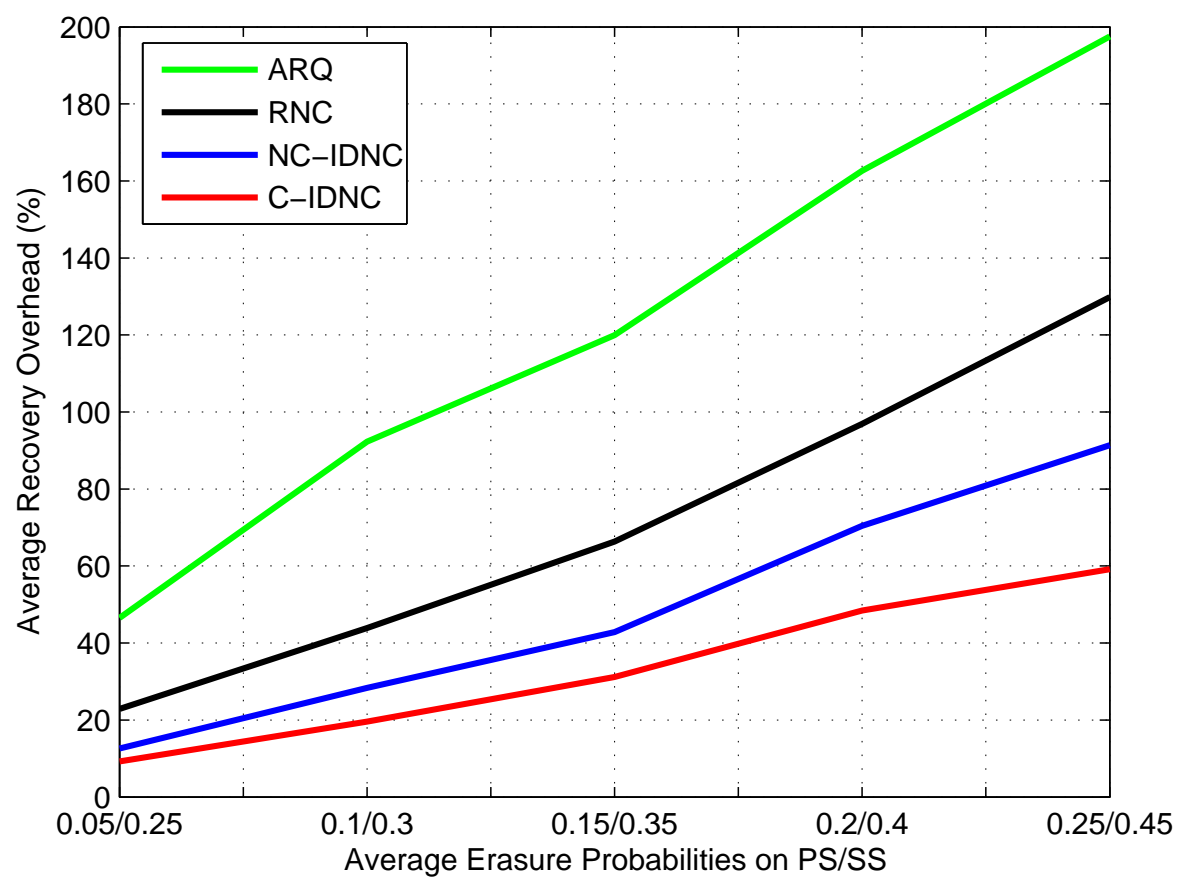

Figure 2.4: Average recovery overhead vs erasure probabilities in PN.

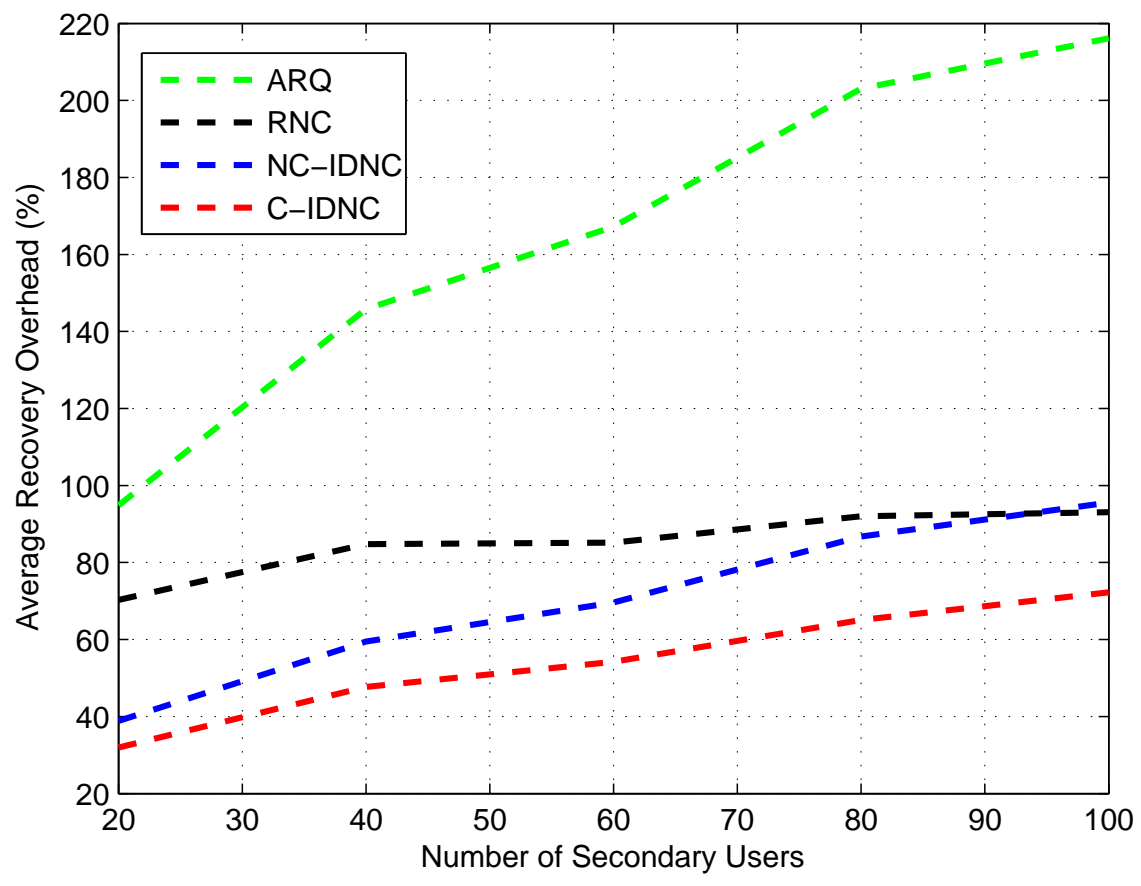

Figure 2.5: Average recovery overhead vs number of SUs in CRN. 


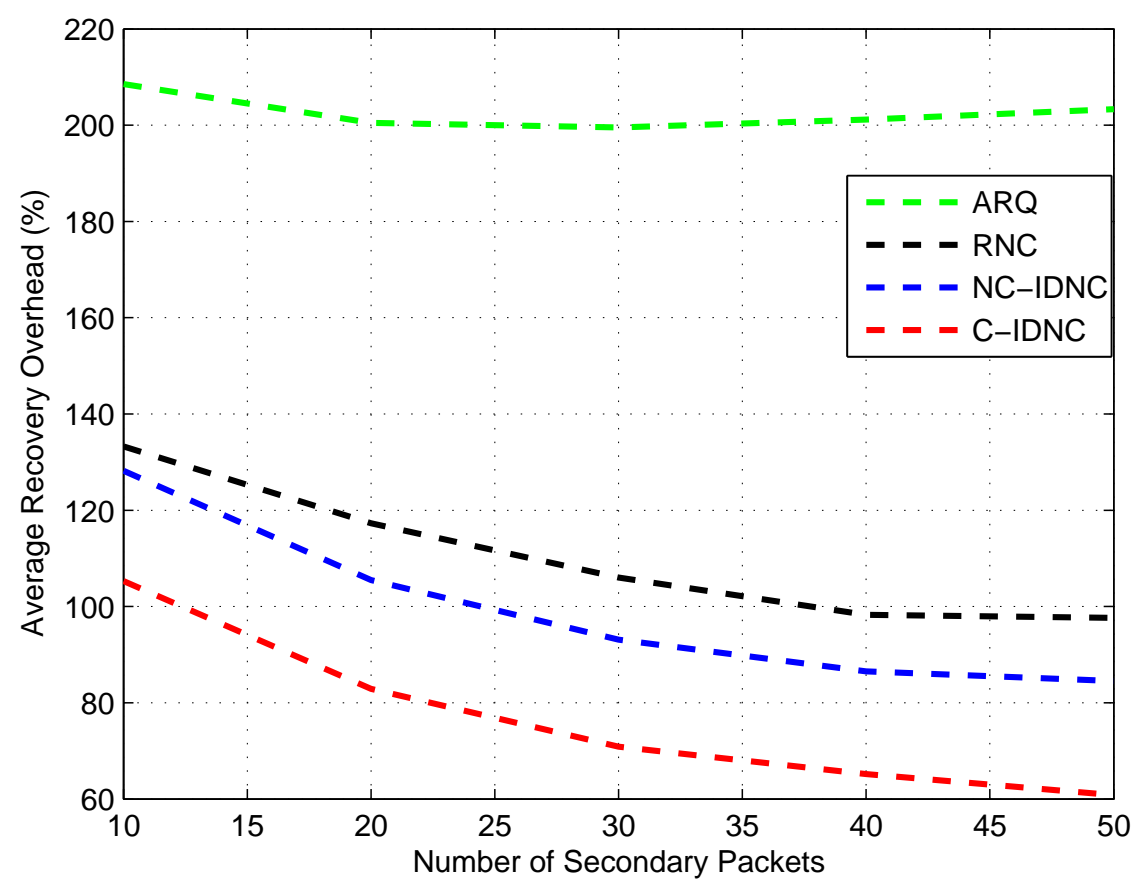

Figure 2.6: Average recovery overhead vs number of SPs in CRN.

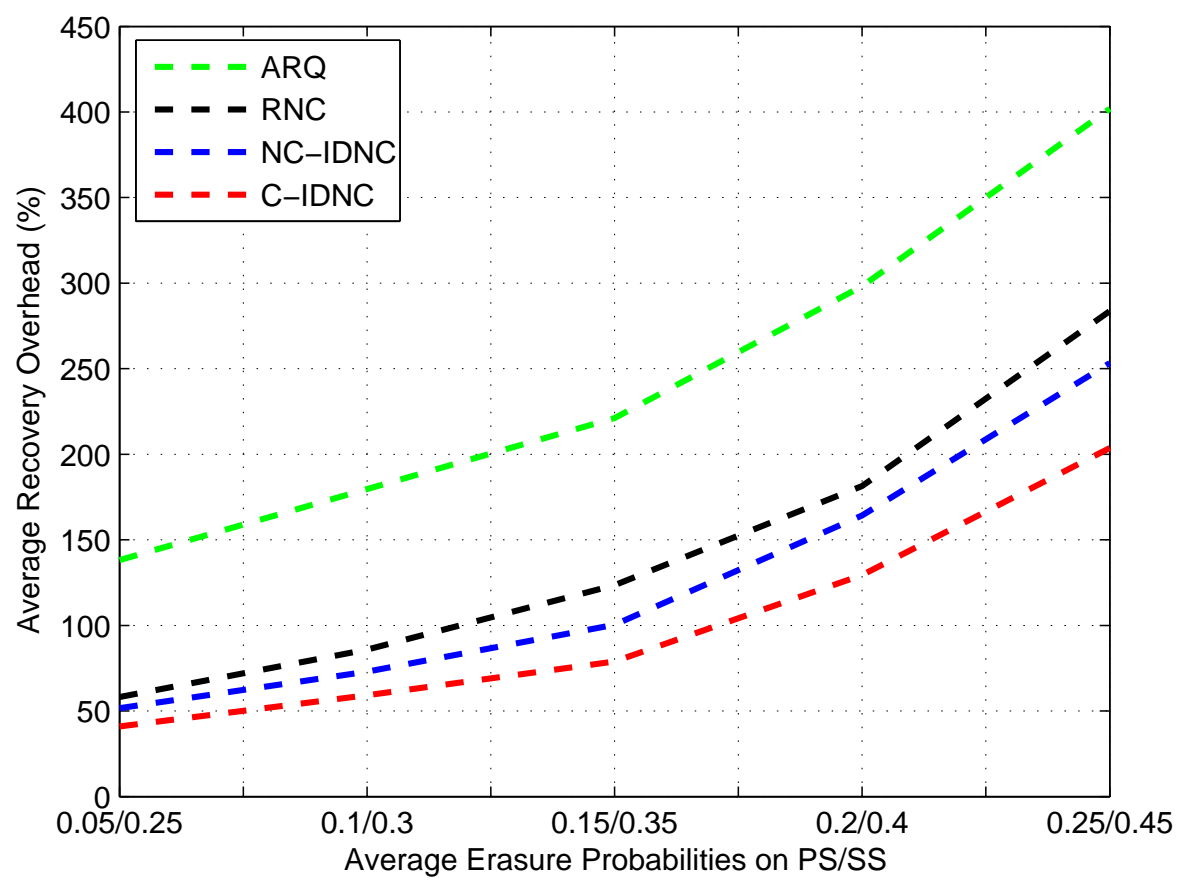

Figure 2.7: Average recovery overhead vs erasure probabilities in CRN. 
scheme by around $12-23 \%$, the RNC scheme by around $23 \%$ and the ARQ scheme by 60-160\% (depending on the metric).

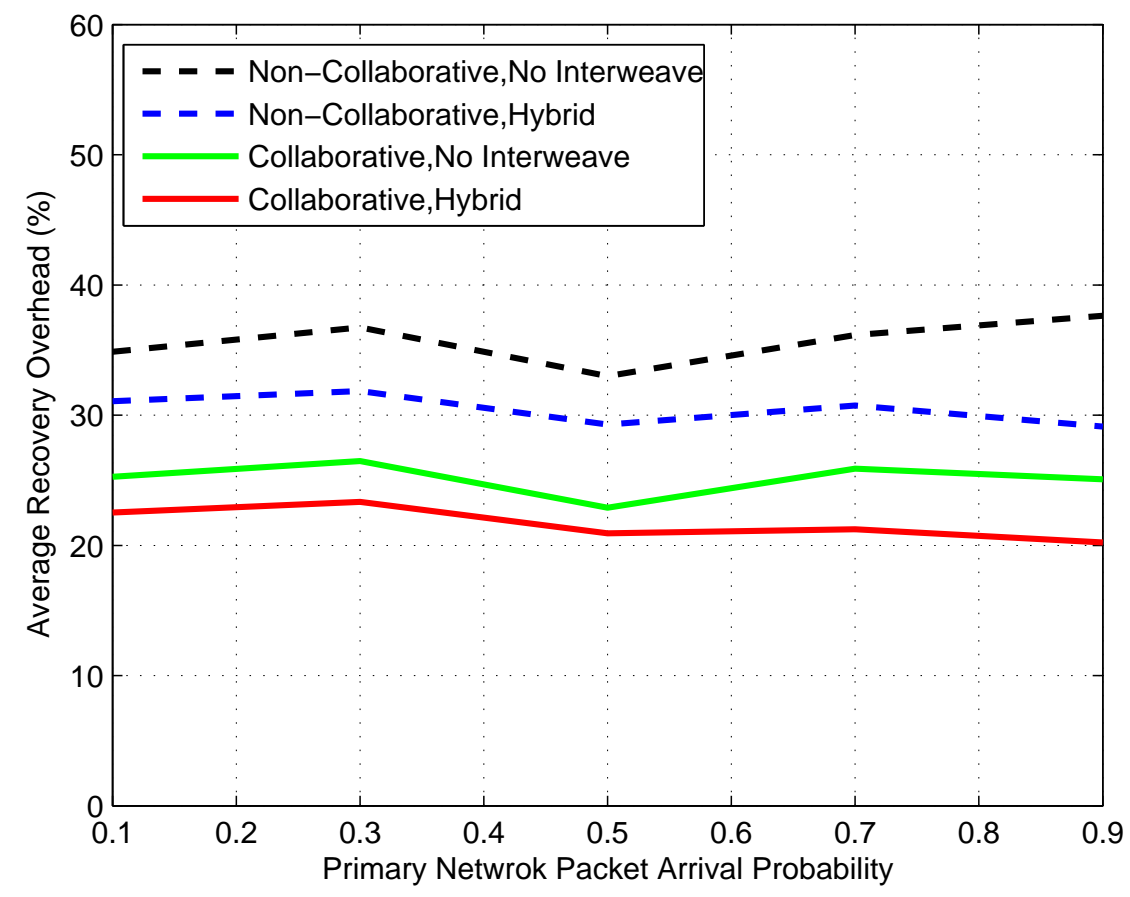

Figure 2.8: Comparing hybrid to no-interweave based systems in PN.

Figures 2.8 and 2.9 show a comparison between the performance of collaborative and non-collaborative schemes in both a hybrid and a no-interweave based system for different primary packet probabilities of arrival. Figure 2.8 shows that the collaborative scheme outperforms the non-collaborative scheme by around $12 \%$ regardless of the probability of arrival. This is expected since the PN's performance shouldn't neither be affected by the type of system utilized nor by the probability of arrival. Figure 2.9 on the other hand depicts how the $\mathrm{SN}$ is affected by both the type of system utilized and the primary packet probability of arrival. Two observations can be made. The first is that as the probability of arrival of primary packets increases, the hybrid system's performance approaches that of the no-interweave system. This is expected since as the probability increases, the CBS will have less opportunities to 


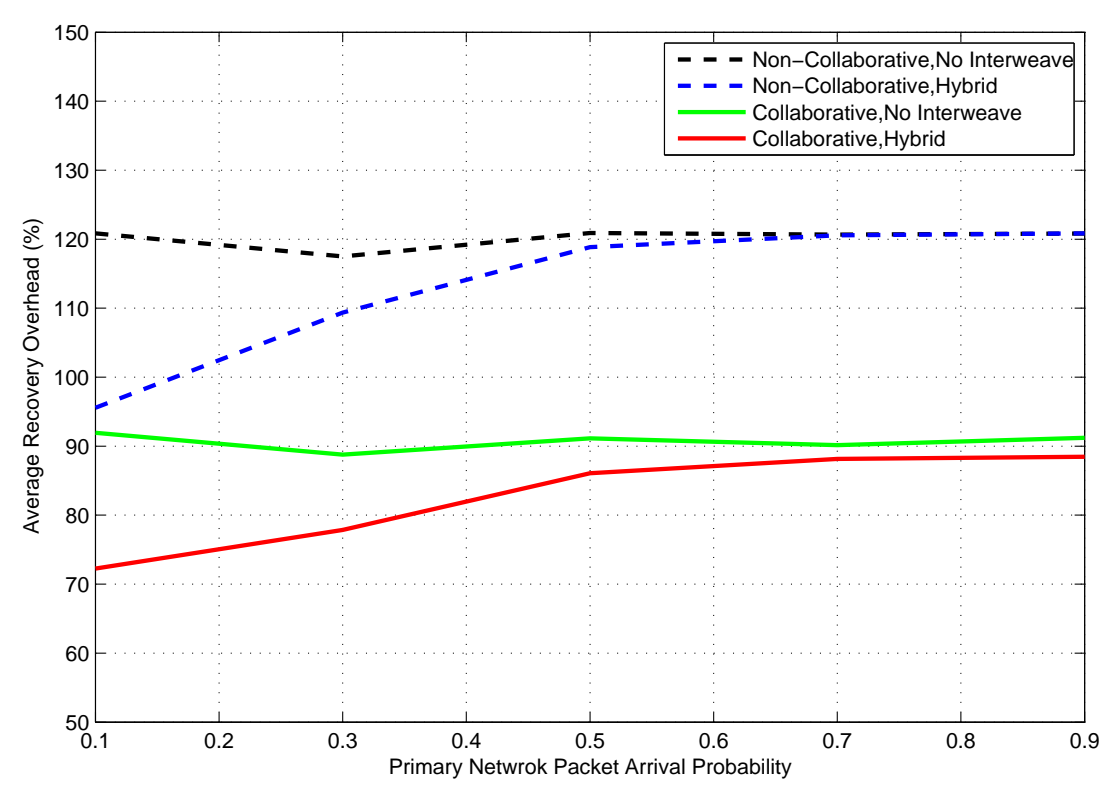

Figure 2.9: Comparing hybrid to no-interweave based systems in CRN.

transmit at higher power and will be in underlay mode for longer time and thus it will perform same as an underlay based system. The other observation is that as the probability of arrival increases, the gap between collaborative and non-collaborative schemes increases. This can be explained by the fact that as the probability of arrival increases, there is more opportunities for the PN and CRN to collaborate, thus making the collaborative scheme more sensible for higher probabilities of arrival. 


\section{Chapter 3}

\section{Effect of Fractional Cooperation in}

\section{Collaborative Multi-Layer Network}

Coding in Multi-Cell Cognitive

\section{Radio Networks}

\subsection{Previous Work}

Fractional cooperation is another spectrum shaping technique suggested to be employed in a CRN setting. Fractional cooperation is defined to be the low-complexity and decentralized wireless networking protocol in which nodes are able to dynamically dedicate a fraction of their resources and commit to forwarding, and where sources can use more than one relay to convey information to the destination [44]. Experimental results show that the protocol can sustain adequate throughput when nodes suddenly enter and leave, making it ideal for dynamically changing systems. Furthermore, the redundancy of information in the network makes it robust to noisy channel conditions. Moreover, when adding a low-complexity coding scheme to fractional cooperation, the system becomes more robust and improves the performance without 
adding any complexity at the receiver's side [45]. This is particularly helpful in wireless sensor networks which have both complexity and energy constraints to adhere to. Fractional cooperation has also proved to be beneficial in femtocell networks [46]. Simulation results show that large gains are possible in typical cellular-style architectures. Furthermore, FC can also be used to determine an upper bound on the max-min rate with a close to optimal performance in a multi-source cooperative network. Simulation results show that for a specific number of sources, there is an optimal number of relays that maximizes the max-min rate [47].

\subsection{System Model and Multi-Layer IDNC Graph and Algorithm}

In our work, we say that fractional cooperation is applied whenever the CRN stops whatever it is doing (either transmitting or recovering) and dedicates all of its resources to help the PN recover its lost packets faster so that the CRN can have a longer time window in which to operate in interweave mode (i.e transmit at higher power). This means that when there is a decision to employ fractional cooperation, the CBS will use the same priorities as the PBS when searching for the maximal weight clique to be transmitted.

The same system model described in chapter 2 is adopted here. We have collocated $\mathrm{PN}$ and CRN cells in a multi-cell PN and multi-cell CRN environment. Each cell consists of a base station and a set of $M_{(i)}$ users (PBS and $M_{P}$ for the PN and CBS and $M_{S}$ for the CRN respectively). A subchannel reuse factor of less than 1 is employed by both networks, indicating that different subchannels are used by adjacent cells. CRN uses the PN subchannel when PN is silent (system is in hybrid mode) and a different subchannel when PN is active (system is in underlay mode). The assumption of perfect sensing is still valid and applicable. Each base station 
broadcasts a set of $N_{(j)}$ packets ( $N_{P}$ primary packets and $N_{S}$ secondary packets). Each subchannel is characterized by its own erasure probability $\left(p_{i}^{(p)}\right.$ is the erasure probability for primary user $i$ and $p_{i}^{(s)}$ is erasure probability for secondary user $i$ ).

$p_{i}^{(p)}$ is lower than $p_{i}^{(s)}$ since the PBS transmits at higher power than the CBS when both networks are active. However, when the PBS is silent, the erasure probability for the secondary user $i$ will become $p_{i}^{(p)}$ since the CBS will be transmitting at higher power.

We employ the same multi-layer IDNC graph and algorithm described in chapter 2. The subgraphs (target and auxiliary subgraphs for each network) are constructed using the basics of graph theory. We apply the same maximum weight clique selection algorithm. The clique selection algorithm is firstly applied to the target subgraph of the respective network since it represents the actual set of missing packets. Then it is extended to the auxiliary subgraph of the respective network. Then the clique selection algorithm is further extended into the target subgraph of the other network and finally into the auxiliary subgraph of the other network. This priority-based multi-layer clique selection algorithm guarantees identifying the same set of packets that would have been sent if there was no collaboration and thus it ensures that each network's performance does not deteriorate while helping the other network whenever it is possible.

\subsection{Proposed IDNC-based Packet Recovery Algo- rithm}

The proposed algorithm runs on seven different phases based on the state of the two networks: $\circ$ Phase 0: PN sends - CRN sends:

Both the PN and the CRN send new frames of uncoded source packets in their initial transmission phase. No collaboration is needed in this phase. However, the 
PBS and CBS as well as all the PUs and SUs overhear each other's packets to use them in later phases. Moreover, a temporary variable ("temp") saves the current state of the CRN so that it can be used in case of FC (if temp $=0 \Rightarrow$ CRN should be recovering packets, if temp $=1 \Rightarrow \mathrm{CRN}$ should be sending new packets). Due to the fact that the networks do not need to be synchronized, this phase can move to one of four phases as follows:

- Both networks finish sending the new frames simultaneously:

- If there is a decision to apply FC by $\mathrm{CRN} \Rightarrow$ Phase 6 and temp $=0$.

- If there is no decision to apply $\mathrm{FC}$ by $\mathrm{CRN} \Rightarrow$ Phase 1.

- Only PN finishes sending its new frame:

- If there is a decision to apply $\mathrm{FC}$ by $\mathrm{CRN} \Rightarrow$ Phase 6 and temp $=1$.

- If there is no decision to apply FC by CRN $\Rightarrow$ Phase 3.

- Only CRN finishes sending its new frame $\Rightarrow$ Phase 2.

\section{○ Phase 1: PN recovers - CRN recovers:}

Both networks recover the lost packets of its users. The multilayer IDNC graph is built and prioritized as shown in the example shown in Figure 2.1 by each of the base-stations. They then generate their coded packets independently based on their priorities and send them over their respective subchannels. The collaboration in recovery can aid both networks in decreasing their recovery overhead. The graph is updated when the feedback is received. This procedure is repeated until one of three events occurs:

- Both networks finish recovery simultaneously:

- If there is a new arrival on $\mathrm{PN} \Rightarrow$ Phase 0.

- If there is no new arrival on $\mathrm{PN} \Rightarrow$ Phase 4. 
- Only PN finishes packet recovery:

- If there is a new arrival on $\mathrm{PN} \Rightarrow$ Phase 2 .

- If there is no new arrival on PN $\Rightarrow$ Phase 5 .

- Only CRN finishes packet recovery $\Rightarrow$ Phase 3 .

○ Phase 2: PN sends - CRN recovers:

A new frame of packets is sent uncoded on the primary subchannel by the PBS which does not help the CRN. In the mean time, the CRN uses the multilayer IDNC algorithm to recover its packets as well as those of the PN. This permits the PUs to receive new packets on the primary subchannel while simultaneously recovering their lost packets on the secondary subchannel. This will significantly reduce the PN's recovery phase and overhead. This is repeated until one of the following three events occurs:

- PN finishes sending its new frame while CRN simultaneously finishes packet recovery:

- If there is a decision to apply FC by $\mathrm{CRN} \Rightarrow$ Phase 6 and temp $=1$.

- If there is no decision to apply FC by $\mathrm{CRN} \Rightarrow$ Phase 3 .

- PN finishes sending its new frame while CRN is still recovering:

- If there is a decision to apply FC by $\mathrm{CRN} \Rightarrow$ Phase 6 and temp $=0$.

- If there is no decision to apply FC by $\mathrm{CRN} \Rightarrow$ Phase 1.

- $\mathrm{CRN}$ finishes packet recovery while PN is still sending its new frame $\Rightarrow$ Phase 0.

○ Phase 3: PN recovers - CRN sends:

This is the complete opposite of phase 2. During this phase, a new frame of packets 
is sent by the CBS, which does not help the PN, over the secondary subchannel. But the CBS as well as the SUs still buffer the overheard packets that the PN is still trying to recover. In the mean time, the PN uses the multilayer IDNC algorithm to recover its packets on the primary subchannel as well as simultaneously hearing the new packets of the CRN. The PBS updates the overhearing information of the packet in its prioritized multi-layer IDNC graph after each new packet transmission. This permits the SUs to receive new packets on the secondary subchannel while simultaneously recovering their lost packets on the primary subchannel. This will significantly reduce the CRN's recovery phase and overhead. This procedure is repeated until one of three events occurs:

- CRN finishes sending its new frame while PN simultaneously finishes packet recovery

- If there is a new arrival on $\mathrm{PN} \Rightarrow$ Phase 2 .

- If there is no new arrival on $\mathrm{PN} \Rightarrow$ Phase 5.

- PN finishes packet recovery while CRN is still sending its new frame

- If there is a new arrival on $\mathrm{PN} \Rightarrow$ Phase 0 .

- If there is no new arrival on $\mathrm{PN} \Rightarrow$ Phase 4.

- $\mathrm{CRN}$ finishes sending its new frame while PN is still recovering $\Rightarrow$ Phase 1.

\section{$\circ$ Phase 4: PN silent - CRN sends:}

In this phase, the CBS takes advantage of the fact that there are no packets being sent to the PUs by the PBS. This allows it to transmit on the primary channel with a higher power without fear of causing interference at the PUs. This helps in decreasing recovery overhead as transmitting with higher power results in a lower error probability. The CBS is constantly sensing whether there is a new batch of 
packets that will be transmitted by the PBS. The system can move from this phase to any of the following three phases depending on the following events:

- CRN finishes sending its new frame and PN simultaneously has a new arrival $\Rightarrow$ Phase 2 .

- CRN finishes sending its new frame and PN has no new arrival $\Rightarrow$ Phase 5 .

- $\mathrm{CRN}$ is still sending its new frame and PN simultaneously has a new arrival $\Rightarrow$ Phase 0.

\section{$\circ$ Phase 5: PN silent - CRN recovers:}

This phase is very similar to Phase 4 in that the CBS sends on the primary subchannel with high power and less erasures due to the silence of the PBS. The only difference is that the $\mathrm{CRN}$ is recovering its packets and not sending new uncoded packets as in Phase 4. Again, depending on the following events, the system can move to one of the following three phases:

- $\mathrm{CRN}$ finishes packet recovery and PN simultaneously has a new arrival $\Rightarrow$ Phase 0.

- $\mathrm{CRN}$ finishes packet recovery and PN has no new arrival $\Rightarrow$ Phase 4 .

- $\mathrm{CRN}$ is still recovering packets and PN simultaneously has a new arrival $\Rightarrow$ Phase 2.

\section{○ Phase 6: PN and CRN recover primary packets:}

In this phase, both the $\mathrm{PN}$ and $\mathrm{CRN}$ recover the primary packets. CRN will choose the same priority order as the PN so that it can help the PN recover faster and thus has more time to send new information or recover its own packets using the primary subchannel. The system can move to one of the four following phases based on the following events: 
- Primary packets are completely recovered and there is a new arrival on the PN:

- If temp $=0 \Rightarrow$ Phase 2 .

- If temp $=1 \Rightarrow$ Phase 0 .

- Primary packets are completely recovered and there is no new arrival on the PN:

- If temp $=0 \Rightarrow$ Phase 5 .

- If temp $=1 \Rightarrow$ Phase 4 .

\subsection{Simulation Results}

The performance of all the previously mentioned schemes is studied in this section to fully understand the effect of fractional cooperation in hybrid cognitive radio networks. The average recovery overhead is plotted with respect to the number of users, the number of packets and the erasure probability of each network respectively.

Note that:

- Solid lines are used to characterize the performance of the PN while dashed lines are used to characterize the performance of the CRN.

- Curve fluctuations are due to finite simulation runs.

Figures 3.1, 3.2, and 3.3 show that for the PN (with probability of arrival of 0.5 and a fractional probability of 0.5 ), the collaborative scheme maintains the same difference by outperforming the non collaborative scheme by around $11 \%$, the RNC scheme by around $25 \%$, and the ARQ scheme by around $30-85 \%$ depending on the metric.

The same trend is observed in the performance of the CRN (with probability of arrival of 0.1 and a fractional probability of 0.5 ). Figures 3.4, 3.5 and 3.6 show 


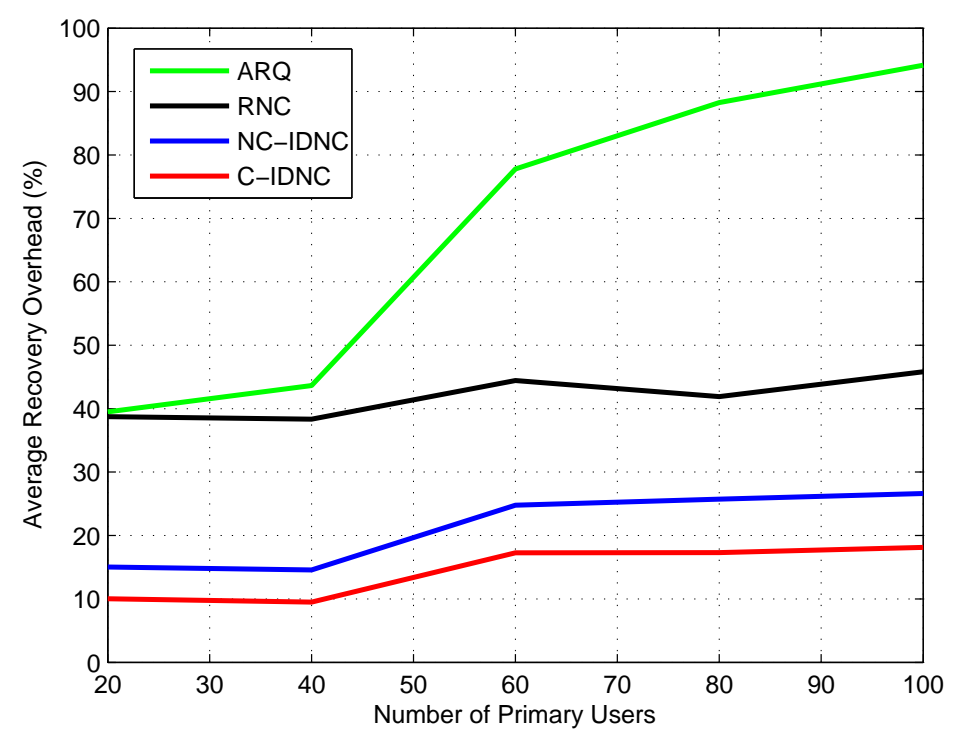

Figure 3.1: Average recovery overhead vs number of PUs in PN with FC.

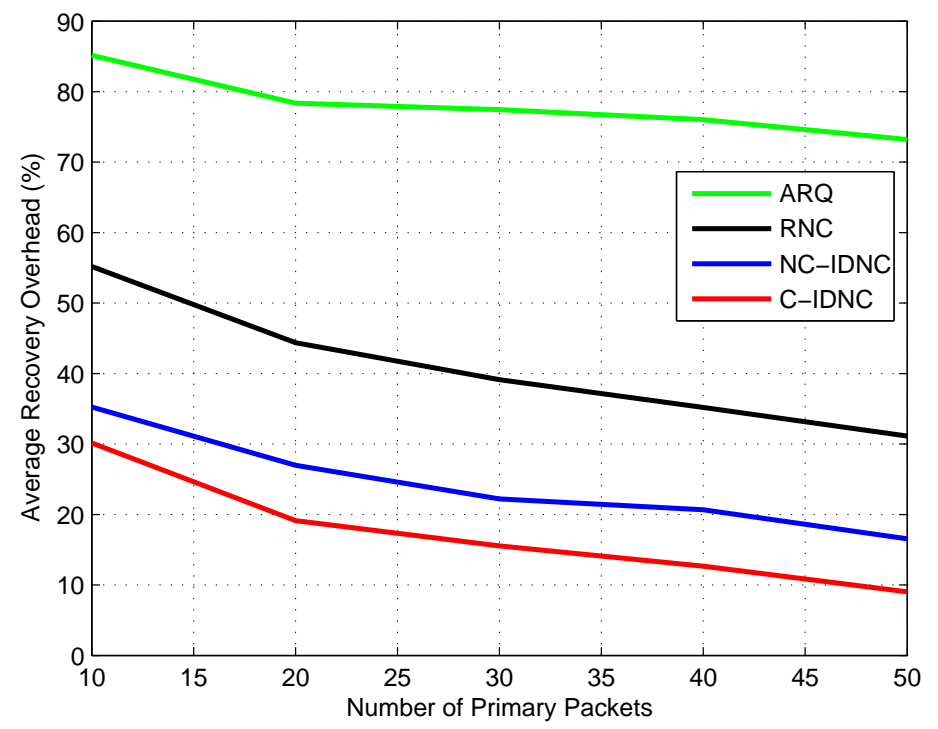

Figure 3.2: Average recovery overhead vs number of PPs in PN with FC. 


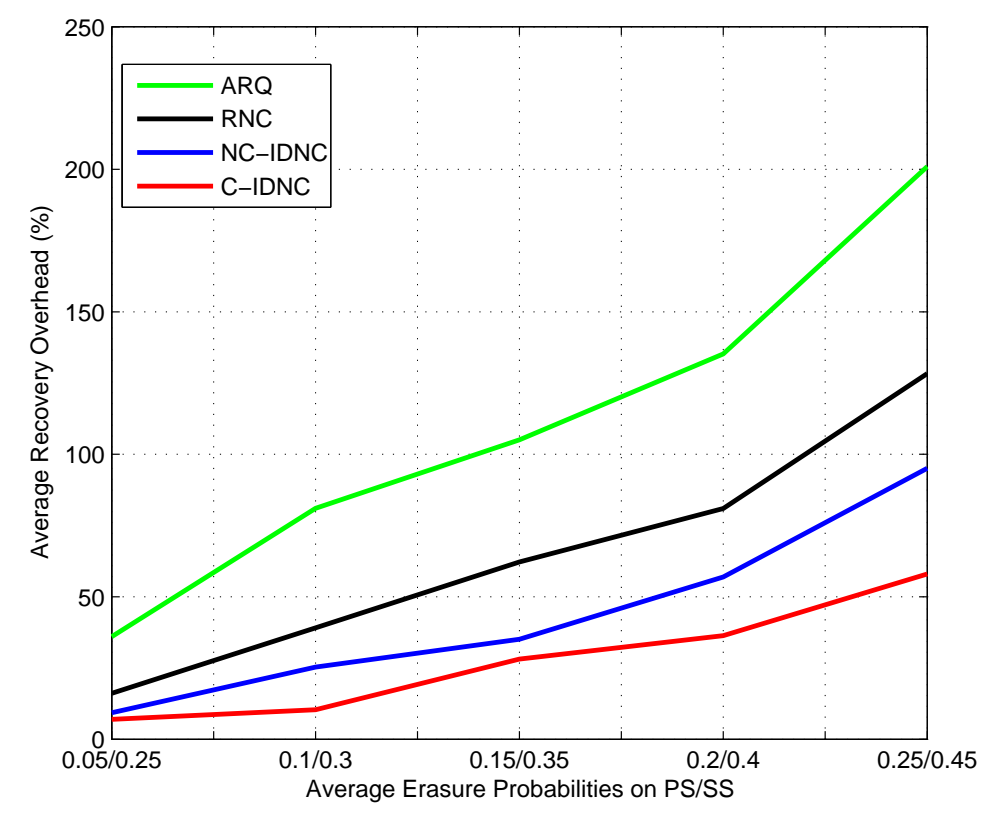

Figure 3.3: Average recovery overhead vs erasure probabilities in PN with FC.

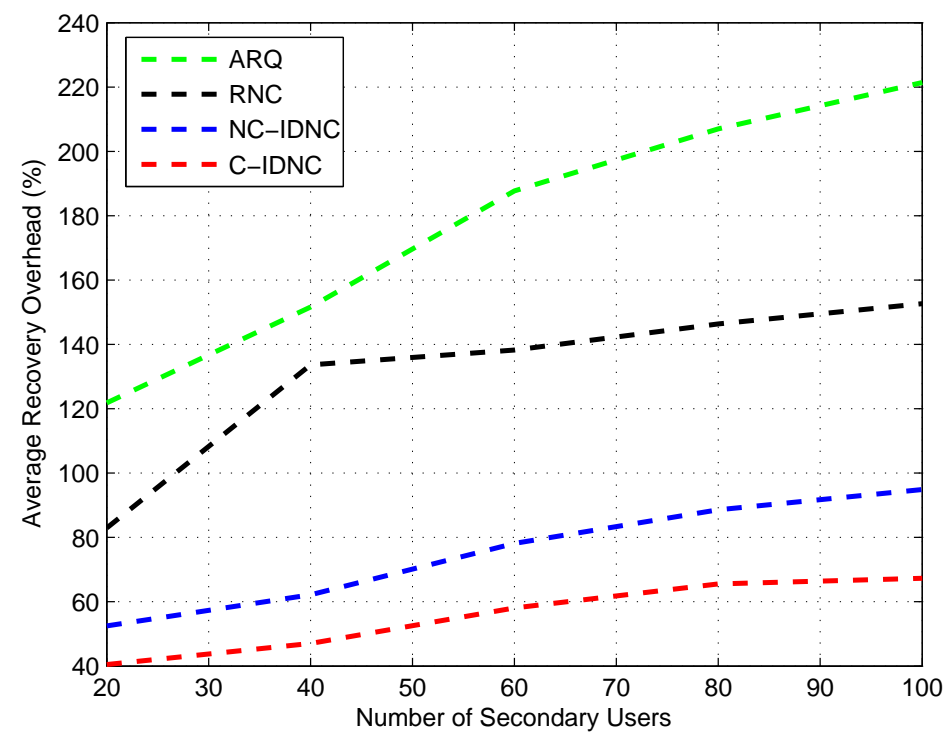

Figure 3.4: Average recovery overhead vs number of SUs in PN with FC. 


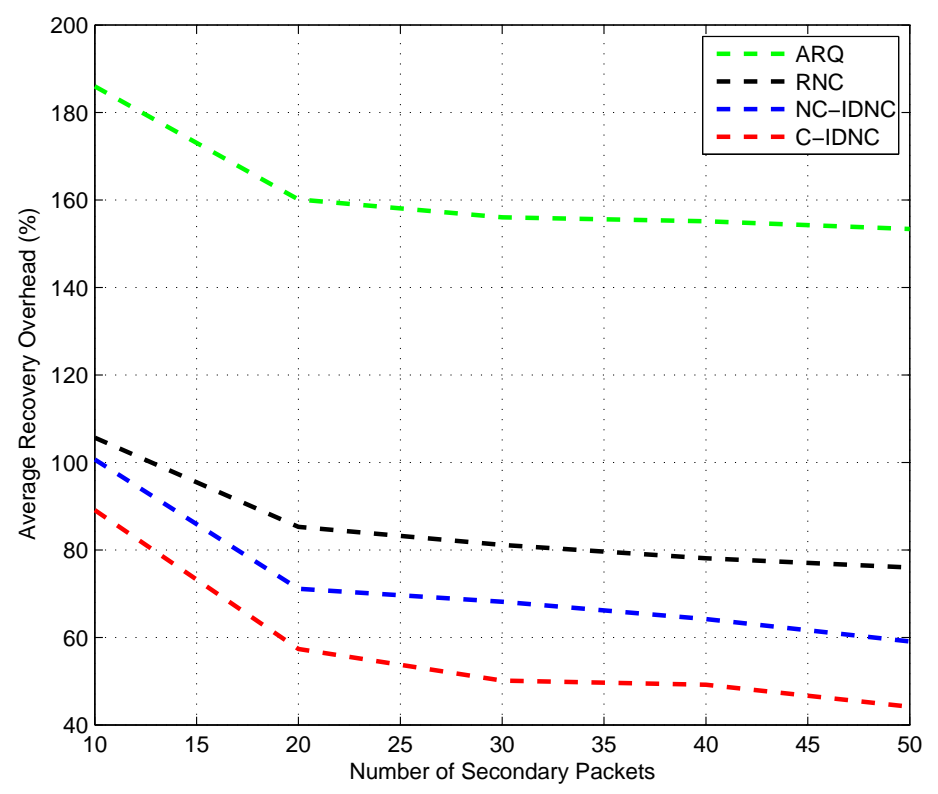

Figure 3.5: Average recovery overhead vs number of SPs in CRN with FC.

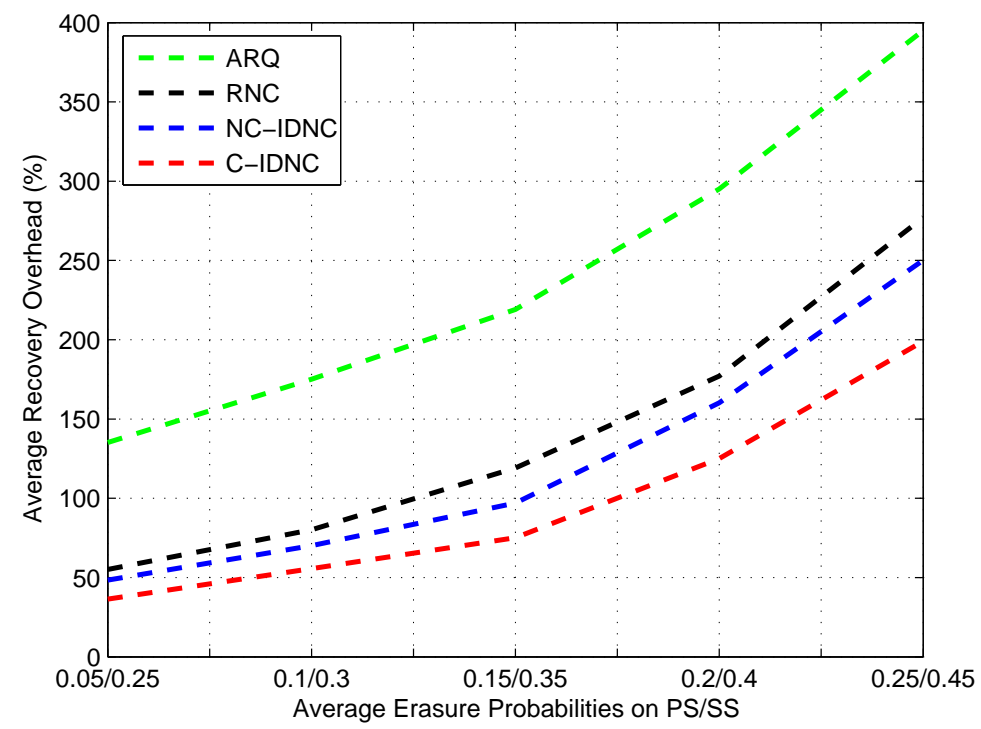

Figure 3.6: Average recovery overhead vs erasure probabilities in CRN with FC. 
that the collaborative scheme outperforms the non-collaborative scheme by around $11-25 \%$, the RNC scheme by around $25 \%$, and the ARQ scheme by around $65-160 \%$.

Moreover, as in chapter 2, simulation results show that as the the probability of arrival of primary packets increases, the performance of the PN remains almost the same while the performance of the CRN deteriorates. This further emphasizes the validity of the results obtained in chapter 2.

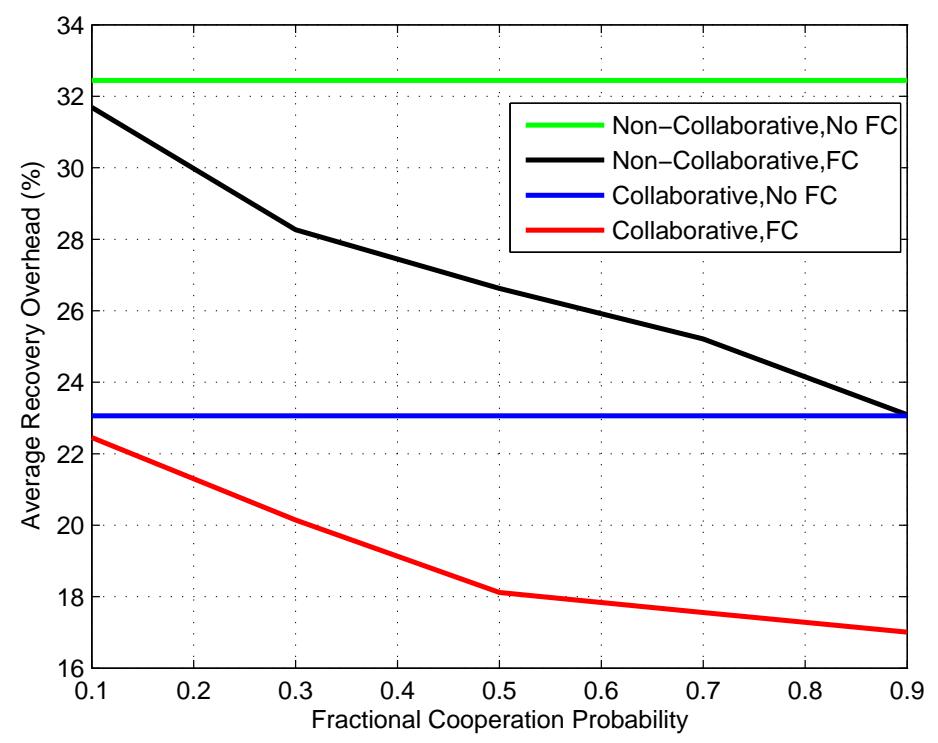

Figure 3.7: Comparing FC-based and Non-FC based systems in PN.

Figures 3.7 and 3.8 show the true effect of utilizing fractional cooperation in a hybrid cognitive radio network. Both the PN and CRN benefit from the use of fractional cooperation. Figure 3.7 shows that the PN performance improves by around $5 \%$ for the collaborative scheme and $7 \%$ for the non-collaborative scheme when a fractional cooperation probability of 0.9 . Figure 3.8 shows that the CRN performance improves by around $10 \%$ for the collaborative scheme and around $5 \%$ for the noncollaborative scheme for the same fractional cooperation probability of 0.9 . The improvement in both networks can be explained in the fact that the PN benefits from 


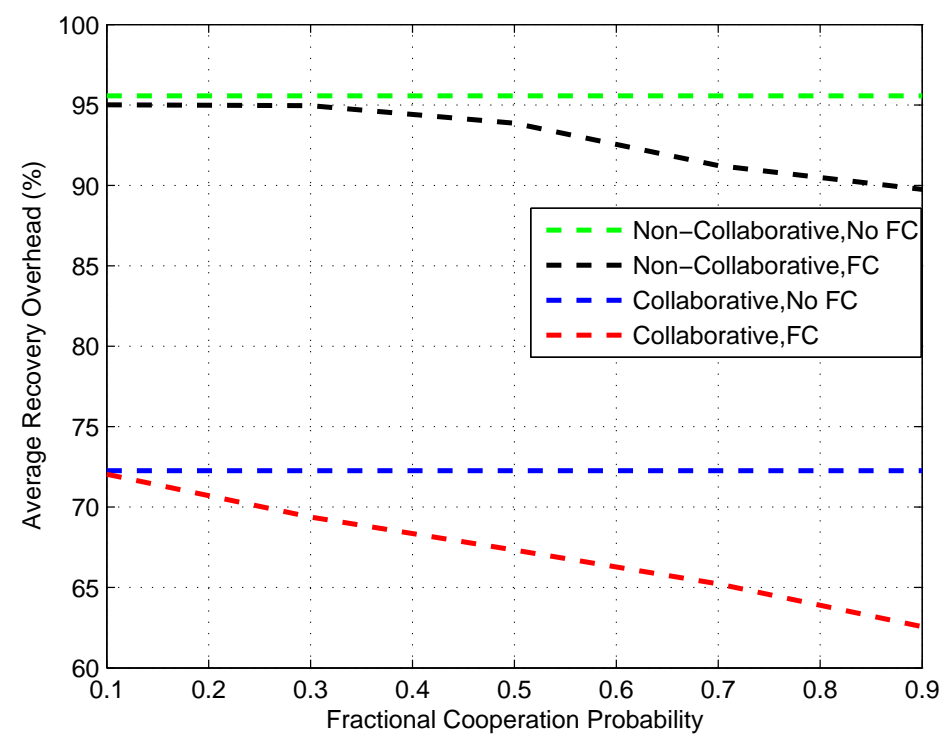

Figure 3.8: Comparing FC-based and Non-FC based systems in CRN.

the CRN sacrifice in which the CRN retransmits only PN coded packets. On the other hand, the CRN benefits from the longer time frame in which the PN is silent to transmit at higher power and therefore reduce its own average recovery overhead. 


\section{Chapter 4}

\section{Concluding Remarks}

\subsection{Summary}

In this thesis, we studied the performance of the collaborative IDNC algorithm for packet recovery in a hybrid cellular cognitive radio network. The algorithm exploits the notion of packet overhearing between the networks to utilize the proposed prioritized multi-layer IDNC approach at both the PBS and CBS in an attempt to improve their throughput performance and recovery overhead. Additional phases representing the hybrid mode were added. In these phases, the CBS utilizes the primary subchannels and transmits at higher power whenever the PBS is silent. Significant improvements are observed on both networks. The performance improvement in the PN is almost constant at around $12 \%$ regardless whether we employ hybrid or underlay system. However, the reduction in recovery overhead in the CRN reaches around $20 \%$ between a hybrid and an underlay system. Moreover, the gap between collaborative and non-collaborative schemes increases from $23 \%$ to about $35 \%$ as the probability increases.

When employing fractional cooperation, further improvements are observed. When using a fractional cooperation probability of 0.9 , the PN performance improves by around $5 \%$ and the CRN performance improves by around $10 \%$. 


\subsection{Future Research Work}

The work done in this thesis can be extended into several directions. Two main extensions can be performed:

- Study the effect of imperfect sensing on the system: We have assumed that we have perfect sensing and that the CRN is able to perfectly detect the spectrum holes. However, we need to study the effect of imperfect sensing and how it affects the performance of both the PN and the CRN.

- Study the performance of the system with a random probability of arrival: We studied the performance when the of primary packet probability of arrival was deterministic. Nonetheless, the performance of the system should be studied whenever the primary packet probability of arrival is varying and random. 


\section{REFERENCES}

[1] S. Sorour, T. Al-Naffouri, and M.-S. Alouini, "Collaborative multi-layer network coding for cellular cognitive radio networks," in Proc. of IEEE International Conference in Communication (ICC'13), Budapest, Hungary, Jun. 2013.

[2] "United states radio spectrum frequency allocations chart as of 2003," Available at http://www.ntia.doc.gov/files/ntia/publications/2003-allochrt.pdf, accessed: 01-04-2014.

[3] "Hong kong frequency allocation chart," Available at http://appsvr.cintec.cuhk. edu.hk/exhibition/project.php?pid=181, accessed: 01-04-2014.

[4] "Mauritian radio frequency spectrum allocation chart," Available at http:// www.icta.mu/images/spectrum.jpg, accessed: 01-04-2014.

[5] A. Cattoni, "Introduction to cognitive radio," PhD Course on "Theory and Practice of Cognitive Radio", Aalborg University, May 2012.

[6] S. Haykin, "Cognitive radio: Brain-empowered wireless communications," IEEE Journal on Selected Areas in Communications, vol. 23, no. 2, pp. 201-220, Feb. 2005.

[7] J. Mitola III and G. Maguire Jr., "Cognitive radio: making software radios more personal," IEEE Personal Communications, vol. 6, no. 4, pp. 13-18, 1999.

[8] P. Popovski, "Information-theoretic aspects of cognitive radio," PhD Course on "Cognitive Radios and Networks: Theory and Practice", Trinity College Dublin, May 2013.

[9] T. Ho and D. S. Lun, "Network coding:an introduction." 
[10] "Report of the spectrum efficiency working group," FCC Spectrum Policy Task Force, Tech. Rep. 02-135, Nov. 2002.

[11] L. Nagurney, "Software defined radio in the electrical and computer engineering curriculum," in Proc. of 39th IEEE Frontiers in Education Conference (FIE '09), Oct. 2009.

[12] J. Mitola III, "Cognitive radio: An integrated agent architecture for software defined radio," Ph.D. dissertation, Royal Institute of Technology (KTH), Stockholm, Sweden, 2000.

[13] J. Wang, M. Ghosh, and K. Challapali, "Emerging cognitive radio applications: A survey," IEEE Communications Magazine, vol. 49, no. 3, pp. 74-81, Mar. 2011.

[14] D. Maldonado, B. Le, A. Hugine, T. Rondeau, and C. Bostian, "Cognitive radio applications to dynamic spectrum allocation: a discussion and an illustrative example," in Proc. of the 1st IEEE International Symposium on New Frontiers in Dynamic Spectrum Access Networks (DySPAN'05), Nov. 2005.

[15] J. A. Ajiboye and A. Y.A., "Cognitive radio networks - a key technology of future wireless communication: Challenges and opportunities," IUP Journal of Telecommunications, vol. 4, no. 4, pp. 37-43, Dec. 2012.

[16] T. Yucek and H. Arslan, "A survey of spectrum sensing algorithms for cognitive radio applications," IEE Communications Surveys Tutorials, vol. 11, no. 1, pp. 116-130, Jan. 2009.

[17] A. Ghasemi and E. Sousa, "Spectrum sensing in cognitive radio networks: requirements, challenges and design trade-offs," IEEE Communications Magazine, vol. 46, no. 4, pp. 32-39, Apr. 2008.

[18] R. Ahlswede, N. Cai, S.-Y. Li, and R. Yeung, "Network information flow," IEEE Transactions on Information Theory, vol. 46, no. 4, pp. 1204-1216, Jul. 2000.

[19] S.-Y. Li and R. Yeung, "Linear codes for network information flow," in Proc. of Information Theory and Networking Workshop, Jun. 1999. 
[20] P. P. Kemei and W. P. Korir, "Emerging network technologies that are likely to become widely available in the future," International Journal of Computer Trends and Technology (IJCTT), vol. 4, no. 5, pp. 1177-1184, May 2013.

[21] A. Le, A. Tehrani, A. Dimakis, and A. Markopoulou, "Instantly decodable network codes for real-time applications," in Proc. of International Symposium on Network Coding (NetCod'13), June 2013.

[22] S.Sorour and S.Valaee, "Minimum broadcast decoding delay for generalized instantly decodable network coding," in Proc. of IEEE Global Communications Conference (GLOBECOM'10), Dec. 2010.

[23] A. Eryilmaz, A. Ozdaglar, M. Medard, and E. Ahmed, "On the delay and throughput gains of coding in unreliable networks," IEEE Transactions on Information Theory, vol. 54, no. 12, pp. 5511-5524, Dec 2008.

[24] H. Hou and H. Li, "The characterization of multi-source multicast with network coding," in Proc. of 14 th IEEE International Conference on Communication Technology (ICCT'12), Nov. 2012.

[25] S. Katti, H. Rahul, W. Hu, D. Katabi, M. Medard, and J. Crowcroft, "Xors in the air: Practical wireless network coding," IEEE/ACM Transactions on Networking,, vol. 16, no. 3, pp. 497-510, Jun. 2008.

[26] M. Yang and Y. Yang, "Peer-to-peer file sharing based on network coding," in Proc. of the 28th International Conference on Distributed Computing Systems (ICDCS '08), Jun. 2008.

[27] Y. Ma, D. I. Kim, and Z. Wu, "Optimization of ofdma-based cellular cognitive radio networks," IEEE Transactions on Communications, vol. 58, no. 8, pp. 2265-2276, Aug. 2010.

[28] J. Jin, H. Xu, and B. Li, "Multicast scheduling with cooperation and network coding in cognitive radio networks," in Proc. of 29th IEEE International Conference on Computer Communications (INFOCOM'10), Mar. 2010. 
[29] S. Wang, Y. Sagduyu, J. Zhang, and J. H. Li, "Spectrum shaping via network coding in cognitive radio networks," in Proc. of 30th IEEE International Conference on Computer Communications (INFOCOM'11), Apr. 2011.

[30] S. Wang, Y. E. Sagduyu, J. Zhang, and J. H. Li, "The impact of induced spectrum predictability via wireless network coding," IEEE Transactions on Vehicular Technology,, vol. 61, no. 2, pp. 758-769, Feb. 2012.

[31] P. Sadeghi, R. Shams, and D. Traskov, "An optimal adaptive network coding schem for minimizing decoding delay in broadcast erasure channels," EURASIP Journal of Wireless Communications and Networking, vol. 2010, pp. 1-14, Apr. 2010.

[32] S. Sorour and S. Valaee, "On minimizing broadcast completion delay for instantly decodable network coding," in Proc. of IEEE International Conference on Communications (ICC'10), May 2010.

[33] — - "Completion delay minimization for instantly decodable network coding with limited feedback," in Proc. of IEEE International Conference on Communications (ICC'11), Jun. 2011.

[34] S.Sorour and S.Valaee, "On densifying coding opportunities in instantly decodable network coding graphs," in Proc. of IEEE International Symposium on Information Theory Proceedings (ISIT'12), Jul. 2012.

[35] J. Sundararajan, D. Shah, and M. Medard, "Online network coding for optimal throughput and delay - the three-receiver case," in Proc. of International Symposium on Information Theory and Its Applications (ISITA'08), Dec. 2008.

[36] L. Keller, E. Drinea, and C. Fragouli, "Online broadcasting with network coding," in Fourth Workshop on Network Coding, Theory and Applications (NetCod'08), Jan. 2008.

[37] E. Drinea, C. Fragouli, and L. Keller, "Delay with network coding and feedback," in Proc. of IEEE International Symposium on Information Theory (ISIT '09), Jun. 2009. 
[38] D. Nguyen, T. Nguyen, and X. Yang, "Multimedia wireless transmission with network coding," in Proc. of 15th International Packet Video Workshop (PV'O'7), Nov. 2007.

[39] D. Nguyen and T. Nguyen, "Network coding-based wireless media transmission using pomdp," in Proc. of 17th International Packet Video Workshop (PV'09), May 2009.

[40] D. Traskov, M. Medard, P. Sadeghi, and R. Koetter, "Joint scheduling and instantaneously decodable network coding," in Proc. of IEE Global Telecommunications Conference (GLOBECOM'09), Nov. 2009.

[41] X. Li, W. C.C., and X. Lin, "On the capacity of immediately-decodable coding schemes for wireless stored-video broadcast with hard deadline constraints," IEEE Journal on Selected Areas in Communications, vol. 29, no. 5, pp. 10941105, May 2011.

[42] C. Zhan, V.-S. Lee, J. Wang, and Y. Xu, "Coding-based data broadcast scheduling in on-demand broadcast," IEEE Transactions on Wireless Communications, vol. 10, no. 11, pp. 3774-3783, Nov. 2011.

[43] J. Howbert and J. Roberts, "The maximum clique problem," Final Project of the course "CSEP 521:Applied Algorithms", Winter 2007.

[44] A. Calce, N. Farsad, and A. Eckford, "An experimental study of fractional cooperation in wireless mesh networks," in Proc. of 22nd IEEE International Symposium on Personal Indoor and Mobile Radio Communications(PIMRC'11), Sep. 2011.

[45] A. Eckford, J. Chu, and R. Adve, "Low complexity and fractional coded cooperation for wireless networks," IEEE Transactions on Wireless Communications,, vol. 7, no. 5, pp. 1917-1929, May 2008.

[46] K. Srinivas, A. Eckford, and R. Adve, "Fractional cooperation in femtocell networks," in Proc. of IEEE Global Communications Conference (GLOBECOM'12), Dec. 2012. 
[47] E. Karamad and R. Adve, "Fractional cooperation and the max-min rate in a multi-source cooperative network," in Proc. of 44th Annual Conference on Information Sciences and Systems (CISS'10), Mar. 2010. 


\section{APPENDICES}

\section{A Papers Submitted and Under Preparation}

- A. Moubayed, S. Sorour, T. Al-Naffouri, and M.-S. Alouini, "Collaborative MultiLayer Network Coding for Hybrid Cellular Cognitive Radio Networks", Submitted to IEEE Global Communications Conference (GLOBECOM'2014).

- A. Moubayed, S. Sorour, T. Al-Naffouri, and M.-S. Alouini, "Fractional Cooperation with Collaborative Multi-Layer Network Coding for Hybrid Cellular Cognitive Radio Networks", Under preparation. 